\title{
NUMERICAL SIMULATIONS OF THE ONSET AND STABILITY OF DYNAMICAL MASS TRANSFER IN BINARIES
}

\author{
Mario C. R. D’Souza, Patrick M. Motl, Joel E. Tohline and Juhan Frank \\ Department of Physics and Astronomy, Louisiana State University, Baton Rouge, LA 70803
}

\begin{abstract}
Hydrodynamical simulations of semi-detached, polytropic binary stars are presented in an effort to study the onset and stability of dynamical mass transfer events. Initial, synchronously rotating equilibrium models are constructed using a self-consistentfield technique and then evolved with an Eulerian hydrodynamics code in a fully selfconsistent manner. We describe code improvements introduced over the past few years that permit us to follow dynamical mass-transfer events through more than 30 orbits. Mass-transfer evolutions are presented for two different initial configurations: A dynamically unstable binary with initial mass ratio (donor/accretor) $q_{0}=1.3$ that leads to a complete merger in $\sim 10$ orbits; and a double-degenerate binary with initial mass ratio $q_{0}=0.5$ that, after some initial unstable growth of mass transfer, tends to separate as the mass-transfer rate levels off.
\end{abstract}

Subject headings: Numerical Hydrodynamics — Interacting binaries: accretion — Masstransfer — Stability — Double White Dwarfs

\section{Introduction}

Most types of binary stars observed today in a long-term interacting phase of their evolution - such as cataclysmic variables, X-ray binaries and contact binaries - and other types that are no longer actively transferring mass, are survivors of a dynamical phase of evolution in a more violent past. Such rapid evolutionary phases accompany the onset of mass transfer that occurs when angular momentum losses or stellar evolutionary processes bring one component of a detached system into contact with its Roche lobe. It is important to investigate the onset and stability of the ensuing mass transfer in order to understand the origin and the evolutionary links between the various types of binary systems that we observe today. The stability of a given binary system upon contact depends on the system's mass ratio, the structure of the donor star, and how mass and angular momentum is redistributed in the binary during the ensuing mass-transfer event. By making reasonable assumptions that encapsulate the essential physics while simplifying the governing equations, analytical treatments can be devised that provide insight into not only the stability, but the long-term evolutionary behavior of mass-transferring, semi-detached binaries (Soberman et al. 1997; Han \& Webbink 1999; Gokhale et al. 2005). But because rapid mass-transfer events, in particular, are intrinsically complex - involving, for example, nonlinear supersonic flows and a gravitational field that changes in a nontrivial way in response to the dynamical exchange of matter between the stars - the rapid phases through which many binaries evolve are not likely to be 
well understood until they are modeled hydrodynamically with the inclusion of as much physics as possible. In this paper, we demonstrate how large-scale numerical, hydrodynamic simulations can be employed to better understand the stability and the long-term evolutionary behavior of binaries as they undergo rapid phases of mass-transfer. Note that in this paper the term "dynamical" is used to mean processes that result in significant changes in the binary parameters and mass-transfer rates in a few orbital periods.

Among several possible mechanisms driving binary evolution, close binaries inevitably lose orbital energy and angular momentum by the emission of gravitational waves. Double degenerate white dwarfs (DWDs) are of particular interest since their expected numbers and rates of emission of gravitational waves are such that they are likely to constitute an important source of background noise for the LISA mission (Hils \& Bender 2000). Details of the time-evolutionary behavior of the gravitational-wave signal that is emitted from any given system depend on the details of mass and angular momentum flow in the binary. Reliable estimates for the rates of emission and the time-frequency characteristics of the emitted signal (the "template") depend on having a good understanding of the above processes. While some progress can be made with analytical prescriptions, theoretically constructed templates are not likely to be useful for the extraction from the background of signals from individual sources until detailed hydrodynamical models can accurately simulate dynamical mass-transfer events in DWDs. Furthermore, it has been realized recently that many of the theoretically predicted and abundant detached DWDs that are driven to contact in a Hubble time, would start mass transfer in a direct impact mode (Marsh et al. 2004). In this mode, the accretion stream hits the accretor's surface and dissipates without forming an accretion disk. Therefore the orbital angular momentum carried by the stream serves to spin up the accretor and is generally not returned by tides to the orbit. One would like to study these processes without the approximations usually adopted for mathematical convenience. Since relatively little is known about these direct impact binaries, it is also of interest to investigate the structure and properties of these flows and explore possible observational signatures.

The above considerations have motivated us to undertake a long-term program that utilizes detailed numerical hydrodynamic simulations to model dynamical phases of mass transfer that can arise when binaries of different types reach a semi-detached state. We have developed a hydrodynamical code that is capable of following such binaries through more than 30 orbits while conserving mass and angular momentum to a high level of precision. As a first step, we studied the evolution of detached polytropic binaries with components of equal and unequal mass (Motl, Tohline \& Frank 2002, hereafter MTF). In the present paper we describe some important improvements that have been made to the code described by MTF and report on our first results of simulations that follow dynamical mass-transfer events in a fully self-consistent manner. We follow the evolution of two different polytropic binary systems whose properties were selected to illustrate the capabilities of our code and to provide some overlap with earlier, related studies. In particular, one of our models has been chosen specifically for comparison with a smooth particle hydrodynamics (SPH) simulation that was presented a decade ago by Rasio \& Shapiro (1995). To our knowledge, this work has not been repeated with an Eulerian code in the intervening years. 
Much of the related numerical simulation work that has preceded ours (see $\S 2.3$ for elaboration) has concentrated on stars having relatively stiff equations of state and on systems that encounter and evolve through a tidal instability. The hydrodynamics of the coalescence, including brief phases of mass-transfer, has been modeled when necessary as a prelude to an almost inevitable merger. Our focus is, instead, on binaries whose components obey a relatively soft equation of state. Our objective is to investigate in more detail the hydrodynamics of mass transfer, the structures arising from this transfer, and the role mass transfer plays during the dynamical phase, sometimes leading the binary closer to a tidal instability and merger, but sometimes saving it from merger and leading it to a slower evolutionary phase.

\section{Theoretical Background}

Our present investigation focuses on the dynamical stability of semi-detached, polytropic binary star systems in circular (or very nearly circular) orbits. A polytrope is a star whose equation of state is governed by the relation,

$$
p=K \rho^{1+1 / n}
$$

where $p$ is pressure, $\rho$ is mass density, $K$ is a constant that defines the specific entropy of the gas, and $n$ is the so-called polytropic index that specifies the degree of compressibility of the gas. Each initial configuration is uniquely defined by specifying: the mass of each star, $M_{\mathrm{d}}$ (the donor) and $M_{\mathrm{a}}$ (the accretor); the orbital separation $a$ (i.e., the distance between the centers of mass of the two stars); the polytropic index $n$; and each star's polytropic constant, $K_{\mathrm{d}}$ and $K_{\mathrm{a}}$. Throughout this presentation, we also will frequently refer to the system mass ratio,

$$
q \equiv \frac{M_{\mathrm{d}}}{M_{\mathrm{a}}}
$$

Although our tools are capable of evolving binaries with any polytropic index and even more general equations of state, here we will only be considering polytropic binaries in which $n=3 / 2$. This particular choice is of interest because key dynamical properties of mass-transferring binary systems that contain low-mass main-sequence (MS) stars or white dwarfs (WD) can be realistically modeled using an $n=3 / 2$ polytropic equation of state, if an appropriate choice is made for the polytropic constants of the two binary components.

For spherically symmetric polytropic stars, the radius of the star $R$ is uniquely determined once the three parameters $n, M$, and $K$ have been specified (cf., Chandrasekhar 1958). For a given polytropic index, the star's mass-radius relationship is uniquely defined as well; specifically,

$$
M=k_{n} R^{(3-n) /(1-n)},
$$

where the proportionality constant $k_{n}$ depends on $K$ and the gravitational constant $G$ through an expression that is determined from a solution of the relevant Lane-Emden equation (cf., Chandrasekhar 1958), for example, $k_{3 / 2}=13.1[K / G]^{3}$. In close binaries, both stars generally are 
rotationally flattened and tidally distorted. Hence, their geometric shape cannot be accurately characterized by a single radius. Nevertheless, an "effective" radius $R_{\mathrm{d}}$ and $R_{\mathrm{a}}$ of the donor and accretor, respectively, can be defined as the radius of a sphere having a volume $V_{\mathrm{d}}$ or $V_{\mathrm{a}}$ that is filled by each distorted star. The proportionality constant that relates the mass to the effective radius is different from the value of $k_{n}$ that holds for spherical polytropes, but the power-law in the mass-radius relationship is approximately the same. For example, if the structure of the donor is well described by a polytrope of index $n=3 / 2$, the donor's effective radius increases as it loses mass according to the relation,

$$
R_{\mathrm{d}} \propto M_{\mathrm{d}}^{-1 / 3}
$$

\subsection{Concepts derived from the point-mass approximation}

For reference we summarize here, using the notation introduced in this paper, some well-known results concerning mass transfer in binaries. For more details, the interested reader may refer to reviews by Paczyński (1971); Rappaport et al. (1982); Hjellming \& Webbink (1987); Verbunt \& Rappaport (1988); King (1988); Soberman et al. (1997); Han \& Webbink (1999); Frank et al. (2002). If, for the moment, we assume that the stars are point masses in a circular orbit, then three parameters $\left(M_{\mathrm{d}}, M_{\mathrm{a}}\right.$, and $\left.a\right)$ are sufficient to uniquely define the binary system's orbital frequency,

$$
\Omega=\left[\frac{G\left(M_{\mathrm{a}}+M_{\mathrm{d}}\right)}{a^{3}}\right]^{1 / 2}
$$

and its total, purely orbital, angular momentum,

$$
J_{\text {tot }}=J_{\text {orb }}=\frac{M_{\mathrm{d}} M_{\mathrm{a}}}{M_{\mathrm{a}}+M_{\mathrm{d}}} a^{2} \Omega=M_{\mathrm{d}} M_{\mathrm{a}}\left(\frac{G a}{M_{\mathrm{a}}+M_{\mathrm{d}}}\right)^{1 / 2} .
$$

For a given total mass, $M_{\text {tot }}=\left(M_{a}+M_{d}\right)$, and angular momentum, $J_{\text {tot }}$, then, it is easy to show that the binary system will have its minimum separation when $M_{\mathrm{d}}=M_{\mathrm{a}}$, that is, when $q=1$. More generally, in a binary whose orbital evolution is driven by systemic angular momentum losses $(\dot{J})_{\text {sys }}$ such as gravitational radiation, the separation will evolve according to

$$
\frac{\dot{a}}{2 a}=\left(\frac{\dot{J}}{J_{\text {orb }}}\right)_{\text {sys }}-\frac{\dot{M}_{\mathrm{d}}}{M_{\mathrm{d}}}(1-q),
$$

where the dots indicate differentiation with respect to time, and we have assumed $\dot{M}_{\mathrm{a}}=-\dot{M}_{\mathrm{d}}$, so that mass is conserved.

For a point-mass binary, the critical Roche surface can be defined analytically in implicit form (e.g., Frank et al. 2002). The effective radius $R_{L}$ of the Roche lobe around the donor is also well-defined. As shown by Eggleton (1983), the ratio $R_{L} / a$ is only a function of the mass ratio, $q$, and it is fairly accurately given by the approximate expression,

$$
\frac{R_{L}}{a} \approx \frac{0.49 q^{2 / 3}}{0.69 q^{2 / 3}+\ln \left(1+q^{1 / 3}\right)} .
$$


A simpler to use, but somewhat cruder approximation, correct to within $6 \%$ in the range $0<q<4$ is due to Pacyński (1971):

$$
\frac{R_{L}}{a} \approx 0.4622\left(\frac{q}{1+q}\right)^{1 / 3} .
$$

With these ideas in mind, if angular momentum and mass are conserved during a mass-transfer event (fully conservative mass transfer), the binary separation is smallest when $q=1$, and the Roche lobe is smallest when $q \approx 5 / 6$. When mass transfer occurs in binaries where the donor may be approximated as an $n=3 / 2$ polytrope, the donor expands when it loses mass according to the radius-mass relationship given by expression (4). The rate of mass transfer in a semi-detached binary in which the donor slightly overfills its Roche lobe depends mainly on the depth of contact which is proportional to $R_{\mathrm{d}}-R_{L}$. Clearly, this rate increases or decreases according to whether the depth of contact itself increases or decreases. With the above assumptions, it is easy to show that

$$
\frac{\dot{R}_{\mathrm{d}}-\dot{R}_{L}}{R_{\mathrm{d}}} \approx \frac{\dot{R}_{\mathrm{d}}}{R_{\mathrm{d}}}-\frac{\dot{R}_{L}}{R_{L}}=\frac{-2 \dot{M}_{\mathrm{d}}}{M_{\mathrm{d}}}\left(q-\frac{2}{3}\right)
$$

Since $\dot{M}_{\mathrm{d}}$ is negative, the depth of contact increases upon fully conservative mass transfer if $q>$ $q_{\text {stable }}=2 / 3$, or decreases if $q<q_{\text {stable }}=2 / 3$. For a more complete discussion of the stability of mass transfer in double-degenerate binaries see Han \& Webbink (1999) (see also references cited therein). It therefore proves instructive to divide our discussion of stability into two broad regimes: $q>2 / 3$ and $q<2 / 3$.

\subsection{Expectations}

$$
\text { 2.2.1. } q>2 / 3
$$

For $q>2 / 3$, the donor expands more rapidly than the Roche lobe, which may actually contract if $q>5 / 6$. The binary separation itself expands if $q<1$ or contracts if $q>1$. Thus, if the donor is initially more massive than the accretor, the orbital separation will decrease with time and the effective radius of the Roche lobe will decrease as well. Since the donor expands upon mass loss, mass transfer will be clearly unstable as the Roche lobe encroaches on the donor, even in the absence of driving. On the other hand, for systems with $q_{\text {stable }}<q \leq 5 / 6$, the donor expands faster upon mass loss than its Roche lobe can expand and the mass transfer rate will still grow with time. Of these systems, if $q$ is initially only slightly above $q_{\text {stable, }}$ unstable mass transfer may proceed until the mass ratio falls below the stability limit. What happens thereafter depends on whether or not the system falls prey to tidal instabilities. If it survives, the mass transfer decays in the absence of driving or evolves toward stable mass transfer if steady driving is present. Systems with an initial mass ratio significantly higher than $q_{\text {stable }}$ are likely to be unstable and merge through a common envelope phase or the donor may be tidally disrupted. 


$$
\text { 2.2.2. } q<2 / 3
$$

When $q<2 / 3$, the donor is initially less massive than the accretor. Hence, in the point-mass approximation as mass is transferred from the donor to the accretor, the orbital separation will increase with time, and $R_{L}$ will increase as well since $q<5 / 6$. This will tend to stabilize the system because the Roche lobe expands away from the original surface of the donor. Even as the donor expands, in the absence of driving the depth of contact will decrease and so will the mass-transfer. Thus in the absence of driving, the mass transfer would ultimately decay to zero, while it would tend to a stable value if driving is present.

Our discussion above assumes that the total angular momentum is purely orbital and that mass transfer is fully conservative. This is adequate provided that the accretion stream has sufficient angular momentum to form a disk around the accretor. In that case tidal torques on the disk will return the angular momentum advected by the stream back to the orbit. As discussed further below, even if mass transfer is fully conservative, when the mass transfer stream directly impacts the accretor and no disk forms, the advected angular momentum spins up the accretor at the expense of the orbital angular momentum reducing $q_{\text {stable }}$ to values below $2 / 3$.

\subsubsection{Direct Impact and Other Finite-Size Effects}

When the finite size of the stars is taken into account, the total angular momentum must be written as the sum of the orbital angular momentum, $J_{\text {orb }}$ as given by Eq. (6), and the spin angular momenta of the donor $J_{\mathrm{d}}$ and the accretor $J_{\mathrm{a}}$. Angular momentum may be exchanged between the orbit and the binary components by advection and by tides. We cite here without proof an equation that describes in an approximate fashion the rate of change of the binary separation under the effects of a systemic loss, direct impact and possible spin evolution due to tidal interactions (see Marsh et al. 2004 or Gokhale et al. 2005 for details):

$$
\frac{\dot{a}}{2 a}=\left(\frac{\dot{J}}{J_{\mathrm{orb}}}\right)_{\mathrm{sys}}-\left(\frac{\dot{J}_{\mathrm{a}}+\dot{J}_{\mathrm{d}}}{J_{\mathrm{orb}}}\right)_{\mathrm{tides}}-\frac{\dot{M}_{\mathrm{d}}}{M_{\mathrm{d}}}\left(1-q-\sqrt{(1+q) r_{h}}\right) .
$$

Following Marsh et al. (2004), the specific angular momentum that is carried by the stream has been expressed in terms of the circularization radius $r_{h} \equiv R_{\text {circ }} / a$, and the term on the right-hand-side of this equation that contains $r_{h}$ accounts for the rate at which angular momentum is transferred by the stream to the accretor. The second term on the right-hand-side represents the effects of purely tidal changes in the spin angular momenta.

As has been emphasized by Marsh \& Steeghs (2002) and Marsh et al. (2004), the consequential loss of orbital angular momentum that accompanies direct impact accretion (compare the last term on the right-hand-sides of Eqs. 7 and 11) acts to destabilize mass transfer. Recent semi-analytic work suggests that this effect alone can reduce the stability limit from $2 / 3$ to a value $q_{\text {stable }} \approx 0.22$ (Gokhale et al. 2005). However, at the onset of mass transfer in a DWD - especially if, initially,

$q \geq q_{\text {stable }}$ - the mass accretion rate $\dot{M}_{a}$ may well exceed the critical rate that yields the Eddington luminosity and the excess mass may be blown away. This effect will act to slightly increase $q_{\text {stable }}$ 
(Han \& Webbink 1999).

In this work, we relax the assumptions required to treat the mass-transfer semi-analytically and instead investigate the stability of $n=3 / 2$ polytropic binaries through direct hydrodynamical simulations. Thus we are able to follow the internal flow of mass and angular momentum via the stream and tides without any of the assumptions usually adopted for mathematical convenience. It should be kept in mind, however, that we do not include in this investigation a self-consistent treatment of thermal relaxation, and the effects of radiative transfer are ignored.

\subsection{Previous, Related, Hydrodynamical Simulations}

The equilibrium and stability of polytropic binary sequences in nearly circular orbits, including the synchronous and irrotational cases, has been comprehensively discussed in a series of papers by Lai, Rasio \& Shapiro (Lai et al. 1993a,b, 1994a,b,c) for systems having various polytropic indexes and mass ratios. Their results were confirmed and extended in a series of papers by Rasio \& Shapiro (1992, 1994, 1995 — henceforth RS92, RS94, RS95 respectively). Using a relaxation method they constructed synchronously rotating equilibrium binaries for various polytropic indexes, mass ratios and initial separations, and followed their hydrodynamic evolution using smoothed-particle hydrodynamics (SPH). In most cases the evolution led to coalescence of the binary, although in one case that was meant to represent binary neutron stars with a stiff equation of state $(n=1 / 2$, $\left.q=q_{\mathrm{RS}}=0.5\right)$ the model binary returned to a new stable configuration after a phase of mass transfer (RS94). Of particular interest to us in the context of this paper are the results of RS95 who investigated the equilibrium and stability properties of binaries with a variety of initial mass ratios $^{1}$ and with polytropic index $n=3 / 2$.

For equal-mass binaries, both MS and WD binaries were represented in RS95 by setting the polytropic constants of the two components to be equal, that is, $K_{\mathrm{d}}=K_{\mathrm{a}}$. A sequence of equilibrium configurations was constructed for a range of separations, $a$, specified by the parameter $r \equiv a / R_{1}$, where $R_{1}$ was the unperturbed radius of the more massive star (primary). These $q=q_{\mathrm{RS}}=1$, equilibrium binaries were then evolved in time using the SPH method and systems with $r \lesssim 2.45$ were found to suffer a dynamical instability. For MS binaries with $q_{\mathrm{RS}}<1$ (polytropic constants were adjusted so as to obtain the MS mass-radius relation) it is the more massive star that overflows its Roche lobe first so, consistent with the expectations discussed above, RS95 found that the resulting mass transfer tended to be unstable. Systems with $q_{\mathrm{RS}} \lesssim 0.4$ (that is, $q=1 / q_{\mathrm{RS}} \gtrsim 2.5$ ) were found by RS95 to be secularly unstable even before the primary star filled its Roche lobe, while a dynamical instability was encountered before the Roche limit in binaries with $q_{\mathrm{RS}} \lesssim 0.25$ (that is, $q=1 / q_{\mathrm{RS}} \gtrsim 4$ ). Finally, $q<1$ systems with WD components (the polytropic constants are set to be equal in order to realize the appropriate WD mass-radius relationship, given here by

\footnotetext{
${ }^{1}$ In RS95, the parameter $q$ was defined as the ratio of the less massive star to the more massive star so that $q \leq 1$ in all cases. In order to avoid confusion, we will use $q_{\mathrm{RS}}$ when referring to the mass-ratios quoted in RS95 then, in order to be consistent with the definition given here in Eq. (2), we will set $q=1 / q_{\mathrm{RS}}$ when the donor is initially more massive than the accretor.
} 
expression 4) were found by RS95 to remain secularly and dynamically stable until $r$ was small enough for the donor to overflow its Roche lobe. The WD binary, $q=0.5$, was found to be unstable to mass transfer and the SPH simulation led to tidal disruption of the donor star and final merger after five orbital periods.

\subsection{In the Context of this Paper}

In $\S \S 4$ and 5 of this paper, we present the results of nine separate nonlinear hydrodynamical simulations that we have conducted in an effort to better understand mass-transfer instabilities in close binary systems and, at the same time, to better understand the capabilities and limitations of our numerical tools. The initial models for these simulations were all unequal-mass, synchronously rotating, $n=3 / 2$ polytropic binaries in circular orbit. As is illustrated in Figure 1, we have examined systems having three different initial mass ratios: $q_{0}=0.843, q_{0}=1.323$, and $q_{0}=0.500$. The first of these $\left(q_{0}=0.843\right)$ was designed to provide a comparison with the benchmark "model UB" evolution that was discussed by MTF. The initial model with $q_{0}=1.323$ (that is, $q_{\mathrm{RS}}=$ $1 / q \approx 0.76)$ was designed to represent a MS binary in which the more massive star makes first contact with the critical Roche surface. The model with $q_{0}=q_{\mathrm{RS}}=0.5$ was designed to represent a low-mass, DWD binary in which the less massive star has the larger radius and therefore makes first contact with its Roche lobe. Results from these new simulations will be compared with the expectations that have been drawn from earlier analytical and semi-analytical investigations, as well as with the nonlinear simulations presented by RS95. A particularly detailed comparison will be made of the $q_{0}=0.5 \mathrm{WD}$ binary system since the parameters defining the initial state of our model were chosen to match as closely as possible the initial state that was investigated by RS95.

\section{Overview of the Computational Tools}

The computational tools used in our present study are the same as those used in MTF except for two modifications, which we describe in detail in $§ 3.1$, below. In summary, we employ: A self-consistent-field (SCF) code to construct each initial binary model; and a three-dimensional, finite-difference hydrodynamics code to evolve each initial model forward in time. Both codes utilize a cylindrical computational grid with $R, \phi$ and $z$ denoting the radial, azimuthal and vertical coordinates, respectively. As stated earlier, our present study is confined to initial states in which the fluid in both stars obeys an $n=3 / 2$ polytropic equation of state, but in general $K_{\mathrm{d}} \neq K_{\mathrm{a}}$. During the hydrodynamical evolutions, the state variables of every fluid element vary in such a way that they follow adiabats having a ratio of specific heats, $\gamma=1+1 / n=5 / 3$.

Our SCF method is based on the iterative technique developed by Hachisu (1986; see also Hachisu et al. 1986). In the past, this technique has been used to construct initial conditions for hydrodynamical studies of the relative stability of equal-mass binary systems (New \& Tohline 1997; Swesty et al. 2000). It also has been used to construct a wide variety of unequal-mass, detached and semidetached binaries (MTF). Here we use the SCF code to build models of synchronously rotating, unequal-mass binaries in a frame that is corotating with the binary's initial orbital frequency $\Omega_{0}$, 
so the two stars are stationary in this frame. The axis of rotation is taken to be parallel to the $z$-axis of the coordinate grid. As input to the code, we must specify: three boundary points on the stars where the mass-density, $\rho$, must vanish; the maximum density $\rho^{\text {max }}$ of each star; and an initial guess for the density distribution, $\rho(R, \phi, z)$. The three boundary points lie along the line that joins the centers of the two stars; they correspond to the inner and outer edges of one star (usually the accretor), and the inner edge for the companion star. The SCF code then iteratively solves for the initial equilibrium configuration through the following steps. For the given density distribution, the resulting Newtonian gravitational potential, $\Phi(R, \phi, z)$, is calculated by solving Poisson's equation (20). Using the value of $\Phi$ at the three boundary points, the code determines the angular frequency of the binary orbit and two integration constants. With these data, the enthalpy $H(R, \phi, z)$ is computed within each star. Using $H$ and the prescribed polytropic equation of state, an improved "guess" for the density distribution is calculated. The iteration repeats until a prescribed convergence criterion is met.

The hydrodynamical code has been designed to solve the equations that govern the flow of inviscid, compressible, self-gravitating fluids in a frame of reference that is rotating with an arbitrarily chosen angular velocity (including zero, in which case it would be an inertial reference frame). Throughout this paper all the simulations have been conducted in a frame that is rotating with the binary system's initial orbital frequency, $\Omega_{0}$, as derived from the SCF code. The primary variables that are evolved forward in time by the hydrodynamical code are the volume-densities of five conserved quantities: the mass density $\rho$, the radial momentum density, $S$, the vertical momentum density, $T$, the angular momentum density, $A$, and an entropy tracer, $\tau$. These quantities are advanced in time via a conservative formulation and an explicit integration of five, first-order hyperbolic partial differential equations (15)-(19): the three components of Euler's equation, and two continuity equations (one for the mass density and one for the entropy tracer). As described in $\S 4$ of MTF, the hydrodynamical time loop consists of applying the source, advection and artificial viscosity operators in a sequence that ensures a nearly second-order accurate time-integration. This is in addition to enforcing the boundary conditions and solving Poisson's equation for the gravitational potential so that the fluid is accelerated in a self-consistent Newtonian gravitational field.

In mass-transfer systems, flow across the L1 Lagrange point is generally expected to be transonic and, thereafter, the mass-transfer stream can quickly acquire Mach numbers $\gtrsim 10$ as it falls toward the accretor. It is therefore not surprising that in the mass-transfer simulations presented here, relatively strong shocks develop as the accretion stream obliquely impacts the surface of the accretor. With this in mind, it is worth reviewing how shocks are handled in our hydrocode (see MTF for more details). In the vicinity of a shock, our (normally) second-order-accurate advection scheme (using Van Leer monotonic interpolation) is reduced to a first-order-accurate scheme to ensure numerical stability, and artificial viscosity is introduced to mediate the shock, spreading the (ideally, infinitesimally thin) shock front over a small number of computational grid zones. (The extra "artificial viscosity" source terms that are introduced into the three components of Euler's equation to accomplish this task are not shown in our $\S 3.2$ summary of the equations, but they are enumerated in MTF.) As a result, momentum is properly conserved across all shocks. Mass is also properly 
conserved, as the equation of continuity (Eq. 18) remains unchanged in the presence of shocks.

Finally, energy will be conserved in an adiabatic shock only if the specific entropy of material increases as it moves through the shock. We have not added source terms to the energy equation in our hydrocode (Eq. 19) to account for this generation of entropy. As a result, material in the accretion stream retains its "pre-shock" specific entropy (it remains on precisely the same $\gamma=5 / 3$ adiabat as it moves from one side of the shock to the other); and post-shock densities and pressures are somewhat higher than would be expected if energy were conserved and the so-called "adiabatic shock jump conditions" (Landau \& Lifschitz 1959) were realized. With this implementation of the energy equation, we are effectively assuming that the post-shock gas instantaneously radiates away the "extra" heat that should have been generated by the shock. While one might argue that it is unreasonable to expect radiation to cool the gas back down to precisely its pre-shock entropy condition, it also seems unreasonable to expect that material immediately behind a realistic accretion shock will not be subject to some amount of radiative cooling. Our handling of the energy equation in the presence of a shock effectively provides a measure of cooling, and does so in a manner that is straightforward to implement numerically and readily reproducible by others who might choose to use our simulations as a benchmark for further work.

\subsection{Recent Modifications to the Hydrodynamical Code}

The task of self-consistently modeling the dynamical evolution of a binary system that is undergoing mass transfer is computationally challenging, as can be understood from the following considerations. The vast majority of material in the hydrodynamical simulation is gravitationally confined within the two stars and is nearly at rest (as viewed from the rotating reference frame). Mild deviations from this state occur in response to the exchange of mass and momentum between the two stars on a timescale that is on the order of the orbital period. At the same time, the dynamics of the mass-accretion stream - which generally will contain supersonic flows that are confined to a relatively small volume of the computational domain — must be accurately resolved and may severely limit the size of the time step that is permitted by the explicit integration scheme. The evolutionary code must maintain the approximate force balance within and between both stars to a high degree of precision in order to permit an accurate treatment of the response of the binary to Roche-lobe overflow. In this context, it is important to emphasize that, as is traditional in the astrophysical fluid dynamics community, our hydrodynamics code has been developed around a conservative formulation of the dynamical equations. This, in itself, ensures that the advection operators preserve the integral of conserved densities to machine precision. However, we are solving a more complex problem where the fluid flow is coupled to the gravitational field through Poisson's equation and, in the presence of the related gravitational source terms, the code, in its entirety, is no longer strictly conservative.

Two modifications to the algorithm described by MTF have been crucial to the recent success of our Roche-lobe overflow simulations. As is detailed in the following two subsections, both have involved subtle modifications to the source terms in Euler's equation. 


\subsubsection{Treatment of Pressure Gradients}

As has been described by MTF, the inertial-frame source terms in Euler's equation were originally written as the gradient of an effective potential that includes the fluid enthalpy. This formulation of the source terms is consistent with the manner in which initial equilibrium structures are constructed in the SCF code. However, when fluid that is tidally stripped from the donor falls directly onto the surface of the accretor, this formulation produces incorrect pressure gradients if the material from the donor has a different specific entropy from the material in the accretor. Accordingly, we have modified the source terms in Euler's equation to properly account for gradients in the fluid's specific entropy. The hydrocode now explicitly calculates $\nabla p$ instead of the product $\rho \nabla H$, as was indicated in the MTF code description. Implications of this change are enumerated in later sections of this paper.

\subsubsection{Center-of-Mass Motion}

By following the evolution of an unequal-mass, detached binary system (model "UB") through just over five orbits, MTF showed that the code conserves angular momentum to an accuracy $\Delta J_{z} / J_{z} \approx 10^{-4}$ per orbit. (See the first row of our Table 3 for a summary of related measurements reported by MTF for model UB.) They also showed that, because linear momentum was not precisely conserved, the center of mass of the binary system slowly wandered away from its initial position (the center of the cylindrical coordinate grid). As Figure 15 of MTF illustrates, by the end of the UB model evolution (just over five orbits), the center of mass of the system had moved a distance $\approx \Delta R / 4$ away from the center, where $\Delta R$ was the radial size of one grid zone. As MTF pointed out, this level of momentum conservation is excellent when compared to the results of other groups who have performed simulations of comparable (or even less) complexity using finite-difference hydrodynamical schemes.

As we embarked upon our present project to model mass transfer in semi-detached binary systems, we were concerned that even very slow motion of the center of mass away from the center of the computational grid might cause problems in evolutions that were followed through significantly more than five orbits. Most importantly, we were concerned that the surface of one or both stars might run into the outer edge of the computational grid as the center of mass of the system wandered farther and farther from the center of the grid. In an effort to confine the center of mass of the system to a region very close to the center of the coordinate grid, we have added a small "artificial" acceleration,

$$
\mathbf{a}^{\mathrm{art}}=\mathbf{e}_{R} a_{R}^{\mathrm{art}}+\mathbf{e}_{\phi} a_{\phi}^{\mathrm{art}}+\mathbf{e}_{z} a_{z}^{\mathrm{art}}
$$

to the source terms of Euler's equation that is designed to counteract the empirically measured rate at which the center of mass was otherwise being accelerated.

As viewed from an inertial reference frame, it is easy to explain how the requisite size and direction of this artificial acceleration vector should be estimated. Simply "measure" the size and direction of the residual (and unphysical) center-of-mass acceleration $\left[\ddot{\mathbf{r}}_{\text {com }}\right]$ inertial at any point in time, then 
set $\mathbf{a}^{\text {art }}=-\left[\ddot{\mathbf{r}}_{\text {com }}\right]_{\text {inertial }}$. Because our simulations have been performed in a frame of reference that is rotating with a constant angular velocity $\boldsymbol{\Omega}=\mathbf{e}_{z} \Omega_{0}$, we have used the standard reference frame transformation,

$$
[\ddot{\mathbf{r}}]_{\text {inertial }}=[\ddot{\mathbf{r}}+2 \boldsymbol{\Omega} \times \dot{\mathbf{r}}+\boldsymbol{\Omega} \times(\boldsymbol{\Omega} \times \mathbf{r})]_{\text {rotating }}
$$

and determined $\mathbf{a}^{\text {art }}$ from the expression,

$$
\begin{aligned}
\mathbf{a}^{\mathrm{art}} & =-\left[\ddot{\mathbf{r}}_{\mathrm{com}}+2 \boldsymbol{\Omega} \times \dot{\mathbf{r}}_{\mathrm{com}}+\boldsymbol{\Omega} \times\left(\boldsymbol{\Omega} \times \mathbf{r}_{\mathrm{com}}\right)\right]_{\mathrm{rotating}} \\
& =-\mathbf{e}_{R}\left[a_{R, \mathrm{com}}-2 \Omega_{0} v_{\phi, \mathrm{com}}-\Omega_{0}^{2} R_{\mathrm{com}}\right]-\mathbf{e}_{\phi}\left[a_{\phi, \mathrm{com}}+2 \Omega_{0} v_{R, \mathrm{com}}\right]-\mathbf{e}_{z}\left[a_{z, \mathrm{com}}\right]
\end{aligned}
$$

where it is understood in the last expression that all components of the center-of-mass position, velocity, and acceleration are measured in the rotating frame. Appendix A details how, in practice, each of the terms in Eq. (14) is evaluated in the hydrodynamic code.

We emphasize that, at each time step, the same vector $\mathbf{a}^{\text {art }}$ was included in the calculation of the acceleration of every fluid element, but a new value of $\mathbf{a}^{\text {art }}$ was determined every integration time step. Although this was not a particularly sophisticated way to correct for center-of-mass motions in our simulations, it proved to be quite successful. For example, Figure 11 shows that the motion of the center of mass of a binary with $q_{0}=0.5$ was confined to within approximately one radial zone of the center of the grid for over 30 orbits for the longest of the three runs presented.

\subsection{Summary of Equations}

In contrast to the form of the three components of Euler's equation that was presented in MTF, our present simulations of mass-transferring binary star systems have utilized the following equations:

$$
\begin{aligned}
\frac{\partial S}{\partial t}+\nabla \cdot(S \boldsymbol{v})= & -\frac{\partial p}{\partial R}-\rho \frac{\partial}{\partial R}\left[\Phi-\frac{1}{2} \Omega_{0}^{2} R^{2}\right]+\frac{A^{2}}{\rho R^{3}}+2 \Omega_{0} \frac{A}{R} \\
& -\rho\left[a_{R, \mathrm{com}}-2 \Omega_{0} v_{\phi, \mathrm{com}}-\Omega_{0}^{2} R_{\mathrm{com}}\right] \\
\frac{\partial T}{\partial t}+\nabla \cdot(T \boldsymbol{v})= & -\frac{\partial p}{\partial z}-\rho \frac{\partial \Phi}{\partial z}-\rho\left[a_{z, \mathrm{com}}\right] \\
\frac{\partial A}{\partial t}+\nabla \cdot(A \boldsymbol{v})= & -\frac{\partial p}{\partial \phi}-\rho \frac{\partial \Phi}{\partial \phi}-2 \Omega_{0} S R-\rho R\left[a_{\phi, \mathrm{com}}+2 \Omega_{0} v_{R, \mathrm{com}}\right]
\end{aligned}
$$

The statements of mass and entropy conservation, and the Poisson equation remain as presented by MTF, namely,

$$
\begin{aligned}
\frac{\partial \rho}{\partial t}+\nabla \cdot(\rho \boldsymbol{v}) & =0, \\
\frac{\partial \tau}{\partial t}+\nabla \cdot(\tau \boldsymbol{v}) & =0, \\
\nabla^{2} \Phi & =4 \pi G \rho .
\end{aligned}
$$




\section{Results of detached binary simulations}

The two hydrocode modifications described in $\S 3.1$ were introduced to improve the accuracy and long-term stability of our simulations so that we would be able to follow the dynamical evolution of close binary systems through many orbits. In order to assess the effects of these changes, we have carefully analyzed the evolution of a binary system whose initial structure matched as closely as possible the unequal-mass, detached binary system with $q_{0}=0.843$ that was discussed in detail by MTF. In MTF, this "benchmark" configuration was referred to as model "UB" (for "unequalmass binary"); we will henceforth refer to our closely related initial configuration as model "Q0.8," reflecting the value of the model's initial mass-ratio. For analysis purposes, this same initial model, Q0.8, was evolved through approximately 5.3 orbits four separate times using slightly different versions of the hydrodynamical code. In what follows we first detail the properties of this initial model, then we describe the results of the four separate evolutions.

Figure $1 a$ shows the critical Roche surface (dashed curve) and the equatorial-plane density contours (solid curves) that trace the surface of the two stars in model Q0.8, as constructed by our SCF code. The inner (L1) Lagrange point on the critical Roche surface is also identified. Table 1 lists the numerical values of various physical parameters that define this initial model. (Where appropriate, the subscript "0" has been used to emphasize that these are initial parameter values derived from the SCF code. All of these physical variables were permitted to vary with time during the course of the hydrodynamic evolutions.) These include parameters for the binary system as a whole $-q_{0}, a_{0}, \Omega_{0}, J_{\text {tot }}$, and $R_{\text {com }}$ (as defined by Eq. A5) - and parameters that define the structure of the individual polytropic stars $-M_{i}, K_{i}, \rho_{i}^{\max }$, the volume occupied by each star $V_{i}$, and the volume of the associated Roche lobe $V_{i}^{\mathrm{RL}}$, where the subscript " $i$ " refers either to the donor or the accretor. We note that, by volume, the less-massive star (destined to become the donor) initially filled $88 \%$ of its Roche lobe and the more-massive star (the accretor) initially filled $72 \%$ of its Roche lobe. We note as well that, initially, the center of mass of this model was positioned almost exactly at the center of the coordinate grid; specifically, relative to the initial binary separation, $R_{\text {com }} / a_{0}=2.35 \times 10^{-6}$ initially.

All of the parameter values in Table 1 are given in units such that $G=R_{\mathrm{SCF}}=\rho_{\mathrm{a}}^{\max }(t=0)=1$,

Table 1. Initial Parameters for Model Q0.8 ${ }^{\dagger}$

\begin{tabular}{lclcc}
\hline \hline $\begin{array}{c}\text { System } \\
\text { Parameter }\end{array}$ & $\begin{array}{c}\text { Initial } \\
\text { SCF Value }\end{array}$ & $\begin{array}{c}\text { Component } \\
\text { Parameter }\end{array}$ & Donor & Accretor \\
\hline$q_{0}$ & 0.843 & $M_{i}$ & 0.0223 & 0.0265 \\
$a_{0}$ & 0.9707 & $\rho_{i}^{\max }$ & 0.8800 & 1.0000 \\
$\Omega_{0}$ & 0.2327 & $K_{i}$ & 0.0387 & 0.0417 \\
$J_{\text {tot }}$ & $2.93 \times 10^{-3}$ & $V_{i}$ & 0.1577 & 0.1634 \\
$R_{\text {com }}$ & $2.28 \times 10^{-6}$ & $V_{i}^{\mathrm{RL}}$ & 0.1788 & 0.2273 \\
\hline
\end{tabular}

\footnotetext{
${ }^{\dagger}$ Parameter values are given here in dimensionless polytropic units. To scale these numbers to other (e.g., cgs) units, see the discussion in Appendix B.
} 
where $R_{\mathrm{SCF}}$ is the outer edge of the cylindrical grid that was used to generate the model in the SCF code. Appendix B provides expressions that can be used to scale the values of these dimensionless parameters to more meaningful (e.g., cgs) units.

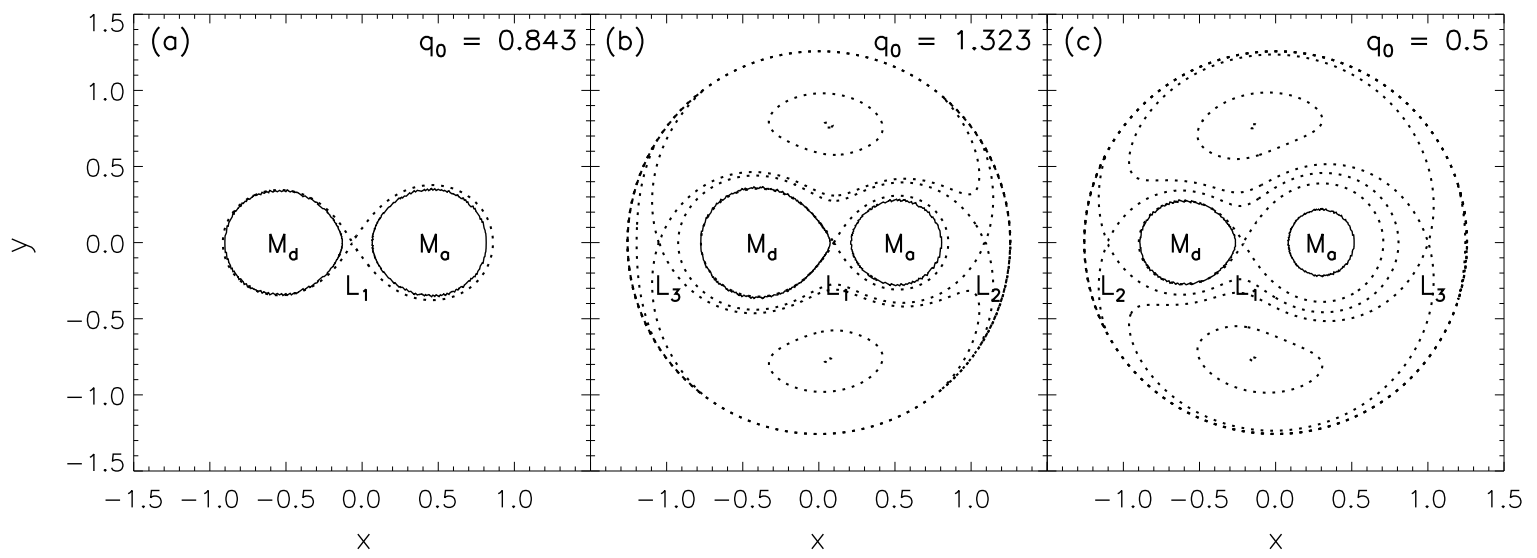

Fig. 1. - A slice through the equatorial-plane is shown for (a) model Q0.8, (b) model Q1.3, and (c) model Q0.5. In each panel, the solid, isodensity contours denote the surfaces of the two stars; they are drawn at a level, $\rho / \rho_{a}^{\max }=10^{-5}$. The "donor" star is located on the left in each panel. The dashed lines trace selected equipotential contours of the self-consistently calculated Roche potential for each model. The outermost circle drawn in panels (b) and (c) identifies the radial edge, $R_{\text {grid }}$, of the computational grid that was used to evolve these initial models hydrodynamically. The location of the inner $\left(L_{1}\right)$ Lagrange point is identified for each system; for models Q1.3 and Q0.5, two additional $\left(L_{2}\right.$ and $L_{3}$ ) Lagrange points are identified because they fall at positions $R<R_{\text {grid }}$.

As is detailed in the first row of Table 2, model Q0.8 was evolved on a uniform grid that had a resolution of $(130,256,98)$ zones in $(R, \phi, z)$, respectively. The hydrocode grid had the same radial extent, $R_{\text {grid }}$, and the same size grid zones, $\Delta z=\Delta R=R_{\text {grid }} /\left(N_{R}-3\right)$ as the grid that was used by the SCF code to construct the initial model, that is, $R_{\text {grid }}=R_{\mathrm{SCF}}=1$ and $\Delta R=7.87 \times 10^{-3}$. As was mentioned above, we evolved model Q0.8 through approximately 5.3 orbits, four separate times, using slightly different versions of the hydrodynamical code. The versions of the code differed, as

Table 2. Computational Grid Parameters

\begin{tabular}{cccccc}
\hline \hline Model & $N_{R}$ & $N_{\phi}$ & $N_{z}$ & $R_{\text {grid }}$ & $\Delta R^{\mathrm{a}}$ \\
\hline Q0.8 & 130 & 256 & 98 & 1.000 & $7.87 \times 10^{-3}$ \\
Q1.3 & 162 & 256 & 98 & 1.251 & $7.87 \times 10^{-3}$ \\
Q0.5 & 162 & 256 & 98 & 1.251 & $7.87 \times 10^{-3}$ \\
\hline
\end{tabular}

\footnotetext{
${ }^{\mathrm{a}}$ In all cases, $\Delta R=R_{\text {grid }} /\left(N_{R}-3\right) ; \Delta z=\Delta R$; and $\Delta \phi=2 \pi / N_{\phi}$.
} 
follows (see $§ 3.1$ for additional clarification):

- Evolution Q0.8-H: The pressure source term in Euler's equation was calculated using $\rho \nabla H$, and no correction was made to limit the motion of the system's center-of-mass. (This is identical to the manner in which source terms were handled in the "benchmark" UB model evolution published by MTF.)

- Evolution Q0.8-P: The pressure source term in Euler's equation was calculated using $\nabla p$, instead of $\rho \nabla H$, but no correction was made to limit the motion of the system's center-ofmass.

- Evolution Q0.8-HC: A very small, artificial acceleration was applied in an attempt to minimize the motion of the system's center-of-mass, but the pressure source term in Euler's equation was calculated using $\rho \nabla H$.

- Evolution Q0.8-PC: The pressure source term in Euler's equation was calculated using $\nabla p$, instead of $\rho \nabla H$, and a very small, artificial acceleration was applied in an attempt to minimize the motion of the system's center-of-mass.

Columns 2 and 3 of Table 3 highlight the differences between these four model evolutions: a "yes" in column 2 means that $\nabla p$ was used in place of $\rho \nabla H$; and a "yes" in column 3 means that $\mathbf{a}^{\text {art }}$ was applied in an effort to correct for the small, but undesired center-of-mass motion.

Although both stars initially filled a large fraction of their respective Roche volumes, neither star was actually in contact with its Roche lobe initially. Hence, the system was not initially susceptible to a mass-transfer instability and nothing was done during these four simulations to artificially excite the instability. As has been explained by MTF, the evolution of this "benchmark" model serves to illustrate how well the fully dynamical hydrocode can preserve the detailed structure of a complex, equilibrium configuration that is close to, but has not exceeded, contact.

Column 4 of Table 3 records the length of time - in units of the initial orbital period, $P_{0}=2 \pi / \Omega_{0}$ - that model Q0.8 was evolved in each of these benchmark simulations. In accordance with our expectations, throughout all four model evolutions, the binary system remained detached, the two stars moved along very nearly circular orbits at a separation $a(t)$ that deviated only slightly from the initial separation, and both stars individually preserved their initial detailed, force-balanced structures to a high degree of precision. The top three panels of Figure 2 show how well key system parameters were preserved throughout all four evolutions: (a) the $z$-component of the total angular momentum $J_{z}(t)$, measured relative to the initial value, $J_{\text {tot }}$; (b) the binary separation $a(t)$, normalized to the initial separation $a_{0}$; and (c) the change in total system mass $\delta M(t) \equiv$ $\left\{M(t)-M_{\text {tot }}\right\}$, measured relative to the initial total mass $M_{\text {tot }}$, where $M(t)$ is the total system mass at time $t$. The separation $a(t)$ reported in all the simulations in this paper is the distance between the centers of mass of the two components. In detail, we identify material belonging to each star by dividing the computational grid into two regions with a plane perpendicular to the binary axis going through the approximate location of the inner Lagrange point. Individual grid cells contribute to the center of mass summation if they are more strongly bound to their respective 
star than the surface layer (a constant density surface). Finally, panel (d) shows the small drift of the center of mass away from the center of the grid as a function of time for the various runs. Note that in all cases the center of mass remained within the first radial cell $\left(\Delta R=7.87 \times 10^{-3}\right)$ and that the drift is significantly reduced by the center of mass corrections for both Q0.8-PC and Q0.8-HC, being almost invisible for the latter.

As the curves in Figure 2 show, over the course of approximately 5 orbits in all four model Q0.8 simulations, the binary lost a very small fraction of its mass, it slowly gained a very small amount of angular momentum and, at the same time, it experienced a slow, very slight secular decay of the orbit. In addition to the slight orbital decay, a small-amplitude oscillation occurs in the binary separation with a period approximately equal to the initial orbital period of the binary. This "epicyclic" motion probably simply reflects that the orbit is not precisely circular, in which case the mean amplitude of the epicyclic oscillation, $(\Delta a / a)_{\text {epicyclic }}$, can be interpreted as the eccentricity of the orbit. The epicyclic motion probably arises because, when the initial model was inserted into the hydrodynamical code, its assigned angular velocity, $\Omega_{0}$, was slightly different from the value that would have been required to place the stars into a perfectly circular orbit. Columns 5 - 8 of Table 3 list, respectively, the secular rate of change per orbit of $J_{z}, a$ and the total system mass $M$, as well as the epicyclic amplitude $(\Delta a / a)_{\text {epicyclic }}$ that resulted from each of our four model Q0.8 simulations; also listed in the first row of the table are the corresponding values from MTF's UB simulation. ${ }^{2}$

In comparing the detailed results of the four Q0.8 evolutions, as summarized in Table 3, we notice that simulations that use the gradient of the pressure conserve angular momentum slightly better (to a level of 5 parts in $10^{5}$, per orbit, instead of 14 parts in $10^{5}$ ) than simulations in which the gradient of the enthalpy was used. At the same time, however, the amplitude of the epicyclic motion was roughly an order of magnitude larger (the orbital eccentricity was $\approx 2 \times 10^{-3}$ instead of $\approx 2 \times 10^{-4}$ ) and the rate of mass loss was approximately twice as large (mass was conserved to a level of 16 parts in $10^{6}$, per orbit, instead of 8 parts in $10^{6}$ ) in the simulations that used the gradient of the pressure. As was noted in $\S 3.1$, expressing the pressure source term

\footnotetext{
${ }^{2}$ We report here a typographical error in the values quoted by MTF for $\Delta M_{1} / M_{\text {tot }}$ and $\Delta M_{2} / M_{\text {tot }}$. The correct values are $\Delta M_{1} / M_{\mathrm{tot}} \approx-3.0 \times 10^{-6}$ and $\Delta M_{2} / M_{\mathrm{tot}} \approx-1.1 \times 10^{-5}$
}

Table 3. Results from Detached Binary Evolutions (Model Q0.8)

\begin{tabular}{lccccccc}
\hline \hline $\begin{array}{c}\text { Simulation } \\
(1)\end{array}$ & $\begin{array}{c}\nabla P \\
(2)\end{array}$ & $\begin{array}{c}\mathrm{COM} \\
(3)\end{array}$ & $\begin{array}{c}t / P_{0} \\
(4)\end{array}$ & $\begin{array}{c}\Delta J_{z} / J_{\text {tot }} \\
(5)\end{array}$ & $\begin{array}{c}(\Delta a / a)_{\text {secular }} \\
(6)\end{array}$ & $\begin{array}{c}\left(\Delta M / M_{\text {tot }}\right) \\
(7)\end{array}$ & $\begin{array}{c}(\Delta a / a)_{\text {epicyclic }} \\
(8)\end{array}$ \\
\hline UB & no & no & 5.178 & $+1.5 \times 10^{-4}$ & $-1.9 \times 10^{-4}$ & $-1.4 \times 10^{-5}$ & $2.2 \times 10^{-4}$ \\
Q0.8-H & no & no & 5.263 & $+1.4 \times 10^{-4}$ & $-1.5 \times 10^{-4}$ & $-8.6 \times 10^{-6}$ & $2.0 \times 10^{-4}$ \\
Q0.8-HC & no & yes & 5.265 & $+1.4 \times 10^{-4}$ & $-1.5 \times 10^{-4}$ & $-8.5 \times 10^{-6}$ & $2.0 \times 10^{-4}$ \\
Q0.8-P & yes & no & 5.269 & $+4.7 \times 10^{-5}$ & $-6.1 \times 10^{-4}$ & $-1.6 \times 10^{-5}$ & $2.2 \times 10^{-3}$ \\
Q0.8-PC & yes & yes & 5.261 & $+4.4 \times 10^{-5}$ & $-4.6 \times 10^{-4}$ & $-1.6 \times 10^{-5}$ & $2.0 \times 10^{-3}$ \\
\hline
\end{tabular}


in Euler's equation in terms of the gradient of the pressure provides the more physically correct description of the evolution of unequal-mass binaries undergoing mass transfer. This may be the reason evolutions Q0.8-P and Q0.8-PC conserved angular momentum better than simulations that used the gradient of the enthalpy. In the context of the difference in the epicyclic amplitude for the two implementations we recall that the SCF code uses the gradient of the enthalpy, rather than the pressure, to construct the initial state. When this initial configuration is placed into the version of the hydrocode that implements the $\rho \nabla H$ source term (evolutions Q0.8-H and Q0.8$\mathrm{HC}$ ), no resultant forces are introduced and the equilibrium of the initial model is therefore well preserved. However, this will not be the case for the version of the hydrocode that evolves the fluid with $\nabla p$. Although, strictly speaking, $\nabla p=\rho \nabla H$ in an isentropic fluid (such as the fluid in either one of the stars in this detached binary), in practice the finite-difference expressions for these two gradients produce slightly different values of the source term. We suspect that the $\nabla p$ implementation introduces very small, but nonzero accelerations throughout the interior of both stars that cause the two stars to oscillate slightly about their own, ideal equilibrium configurations, and that it is the coupling between these stellar oscillations and orbital dynamics of the system as a whole that excites the larger epicyclic motions in evolutions Q0.8-P and Q0.8-PC.

The effect of the center of mass correction was less dramatic than implementing the gradient of the pressure or enthalpy; it slightly improved the conservation of angular momentum and also slightly reduced the amplitude of the epicyclic motion. However, the total mass of the binary, on the scale presented here, remained virtually unchanged for simulations with or without the center of mass correction.

The three principal spurious effects - the slow decay of the orbit, the slow gain of angular momentum and the slow loss of mass from the system - seen in MTF's UB simulation are still present in the simulations presented here. However, depending on the version of hydrodynamical code we choose, our simulations show that the size of these effects can be modified somewhat. The criterion for choosing one version over another rests on the type of binary system we want to simulate. For detached/semi-detached binaries having similar components with respect to the polytropic constant, $\nabla H$ will correctly describe the evolution even if mass transfer occurs in the system. On the other hand if the components have different polytropic constants (i.e., the fluid in the two stars has different specific entropies) then the $\nabla p$ scheme needs to be used, particularly for binary systems that undergo mass-transfer. The disadvantage of using the $\nabla p$ scheme is that a relatively large epicyclic motion is induced in the orbit, and mass is conserved to a slightly lower degree of accuracy than when the $\nabla H$ scheme is utilized. The former can be reduced by modifying the angular velocity to place the initial model in a nearly circular orbit or by "tweaking" the initial state so as to balance the forces which arise from implementing the $\nabla p$ scheme. In all five of the mass-transfer simulations that we present below in $\S 5$, the "PC" version of the hydrocode has been used (consistent with the set of equations that has been summarized above in §3.2) because this version is best suited to follow evolutions through many orbits when the material residing in the donor has a specific entropy that differs from the material initially making up the accretor.

The number recorded in column 7 of the last row of Table 3 provides a reasonable estimate of the 
lowest mass-transfer rate that we will be able to resolve with our existing hydrocode. Specifically, we should expect to only be able to resolve mass-transfer rates $\dot{M}_{d}\left(P_{0} / M_{\text {tot }}\right)>\dot{M}_{\text {min }}=-1.6 \times 10^{-5}$ because, as has just been demonstrated, even a detached binary system like our model Q0.8 slowly loses mass at this rate. In order to accommodate this restriction in our simulations of masstransferring binary systems, we will be required to "drive" the donor into sufficiently deep contact with its Roche lobe that mass transfer proceeds at a rate that exceeds $\dot{M}_{\text {min }}$. For MS Algol-type binaries $\left(M_{\text {tot }} \approx 5 M_{\odot}, P_{0} \approx 3\right.$ days) (Blondin et al. 1995), this limit is equivalent to $\sim 10^{-2} M_{\odot} / \mathrm{yr}$; and for AM CVn-type DWD binaries $\left(M_{\text {tot }} \approx 1 M_{\odot}, P_{0} \approx 0.3 \mathrm{hr}\right.$ ) (Patterson et al. 1993; Nelemans, et al. 2001), this limit is equivalent to $\sim 0.4 M_{\odot} /$ yr. Both of these limits are orders of magnitude larger than the stable mass-transfer rates that are observed in Algol-type or AM CVn-type binary systems. Hence, as we remarked in the introductory section of this paper, we are unable to model long-term, stable evolutionary phases of mass-transfer in such systems. However, during phases of unstable mass-transfer — which are the focus of our present study — the rate of mass-transfer can easily climb to levels above $\dot{M}_{\text {min }}$ and can be satisfactorily modelled with our hydrocode. Note that the minimum resolvable mass transfer $\dot{M}_{\text {min }}$ is on the order of the Eddington critical rate for both white dwarfs and main-sequence donors, but to treat radiative forces is beyond the scope of this paper. Analytic considerations (Han \& Webbink 1999) suggest that the effect of mass loss occurring at super-Eddington mass-transfer rates is stabilizing, thus ignoring the radiative forces at this stage provides a more stringent test of stability. Ideally one would like to be able to follow the evolution with all relevant physical effects included and to resolve realistic mass transfer rates, but this is not possible with the computational resources available today.

\section{Mass-Transfer Simulations}

We now present results from mass-transfer simulations of two semi-detached, $n=3 / 2$ polytropic binary systems with initial mass ratios, $q_{0}=1.323$ and $q_{0}=0.5$; these will henceforth be referred to as models Q1.3 and Q0.5, respectively. Paralleling the information provided in Table 1 for model Q0.8, Tables 4 and 5 list system parameters as well as information about the structure of the component stars for these two initial models, and Figures $1 \mathrm{~b}$ and $1 \mathrm{c}$ illustrate their initial equatorialplane structures. In both panels, the donor is the star on the left which nearly fills its critical Roche surface. The outermost circle in Figures $1 \mathrm{~b}$ and $1 \mathrm{c}$ identifies the edge of the computational grid on which both of these models were evolved. In contrast to our simulations of model Q0.8, and as is summarized in Table 2 , the edge of the hydrocode grid $\left(N_{R}=162 ; R_{\text {grid }}=1.251\right)$ was extended beyond the grid that was used in the SCF code $\left(N_{R}=130 ; R_{\mathrm{SCF}}=1.000\right)$ in order to ensure that the L2 and L3 Lagrange points both were included in the computational domain. Material that flows radially outward across either one of these two saddle points in the effective potential is unlikely to return to either one of the stars on a dynamical timescale, but the dynamical motions of material that lies inside the L2 and L3 locations should be fully included in the hydrodynamical simulations.

These particular models were chosen for this investigation, in part, because their values of $q_{0}$ fall 
into two separate stability regimes, as outlined above in $\S 2.2$. For model Q1.3, $q_{0}>1$ so the binary is expected to be violently unstable to mass transfer. For model Q0.5, $q_{0}$ falls below the value $q_{\text {stable }}=2 / 3$ at which binaries with $n=3 / 2$ polytropic structures should become stable against mass transfer (Han \& Webbink 1999), if the effect of direct-impact accretion is ignored. In addition, the properties of the stars in these two models were specified in such a way that they permit us to examine two distinctly different, but astrophysically interesting evolutionary scenarios: In model Q1.3 (see Table 4 ), the ratio of the effective stellar radii $R_{d} / R_{a}=\left(V_{d} / V_{a}\right)^{1 / 3} \approx M_{d} / M_{a}$, which mimics the mass-radius relationship of MS stars; and in model Q0.5 (see Table 5), $K_{d}=K_{a}$ and $\left(R_{d} / R_{a}\right)^{3}=\left(V_{d} / V_{a}\right) \approx M_{a} / M_{d}$, which represents well the structural properties and mass-radius relationship of stars in a low-mass DWD system. Finally, in the $q<2 / 3$ parameter regime, we specifically selected an initial model with $q_{0}=0.5$ in order to permit a direct comparison with one of the SPH simulations that was reported by RS95.

In both models, as they were generated by the SCF code, the donor star slightly underfills its Roche lobe initially. Specifically, for model Q1.3 (see Table 4$), R_{d} / R_{d}^{\mathrm{RL}}=\left(V_{\mathrm{d}} / V_{\mathrm{d}}^{\mathrm{RL}}\right)^{1 / 3}=0.989$, and for model Q0.5 (see Table 5), $R_{d} / R_{d}^{\mathrm{RL}}=\left(V_{\mathrm{d}} / V_{\mathrm{d}}^{\mathrm{RL}}\right)^{1 / 3}=0.965$. This means that neither model was actually undergoing mass-transfer when it was introduced into the hydrocode. In the context of the SCF technique, it is possible to iteratively refine the converged model configuration to produce semi-detached binaries that come progressively closer to filling their self-consistently determined Roche volume, up to some limiting volume-filling factor $\lesssim 1.00$ that is set by the finite grid resolution of the SCF code. But the SCF technique will not converge to a model where stellar material extends beyond the Roche lobe as this material can not be in hydrostatic equilibrium. Even if the SCF code were able to generate models where the donor star completely fills its critical Roche surface, the models would have little additional practical utility over models, such as Q1.3 and Q0.5, where $R_{d}$ is $97-99 \%$ of $R_{d}^{\mathrm{RL}}$. This is because such models would only be marginally unstable to mass transfer so the time that would be required for $\dot{M}_{d}$ to grow to levels that are of interest (or even resolvable) in the present investigation would be prohibitively long for a timeexplicit hydrodynamics scheme such as ours. It is therefore necessary for us to employ some means of artificially "driving" each binary model into deep enough contact with its Roche surface to ensure that, early in the hydrodynamical evolution, the mass transfer rate climbs to a rate that is resolvable, that is, to a rate $\gtrsim \dot{M}_{\text {min }}$. After this has been achieved, the driving is turned off, except in one case of the Q0.5 runs in which the driving was applied throughout the entire eveolution.

One can imagine several possible mechanisms for driving a binary system into contact. For example, RS95 began with two widely separated, spherical stars and slowly damped the orbital velocity with an artificial friction term until the donor began to lose particles through the inner Lagrange point. This technique is particularly well adapted to the SPH method, as it is free of a computational grid. We instead begin with tidally deformed, rotationally flattened equilibrium models that are already very near contact. To proceed from this point we could simply impart an inward radial kick to the donor, but this would certainly cause the orbit to immediately become noncircular. Alternatively, we could attempt to mimic natural processes by (a) forcing the donor to slowly expand to fill its Roche lobe - a process that will occur in MS binaries as the more 
massive star evolves off the main sequence - or (b) slowly removing orbital angular momentum, thereby forcing the Roche lobe to contract around the donor — as occurs in DWD systems as angular momentum is lost from the system via gravitational radiation. Ideally, the outcome of a given mass-transfer instability should be insensitive to the evolutionary process that has brought the donor star into contact with its critical Roche surface.

In our present investigation, we have explored both of these more natural processes as a means of driving the donor into sufficiently deep contact with its critical Roche surface so that $\dot{M}_{d}$ climbs above $\dot{M}_{\text {min }}$. Of course, because the hydrodynamical evolutions have been followed with an explicit time-integration scheme, we have found it necessary to "drive" the donor into contact at a rate that far exceeds the natural thermal expansion rate of evolving MS stars or the natural rate at which angular momentum is lost from DWD binaries. The rates we have employed are, nevertheless, slow enough that during the early (and generally brief) phase of artificial driving, the two stars individually, as well as the system as a whole, remain very near the initial equilibrium state as generated by the SCF code. Thus, as desired, the artificial driving introduces only secular, rather than dynamical, changes in the system.

In order to initiate mass transfer in model Q0.5 (see §5.2), we drained orbital angular momentum from the system at a rate of $1 \%$ of $J_{\text {tot }}$ per orbit for varying amounts of time in order to achieve varying depths of contact and levels of mass transfer. These simulations will henceforth be collectively referred to as Q0.5-D and individually as Q0.5-Da, -Db, and -Dc in order of increasing driving time (see $\S 5.2$ for details). For model Q1.3 (see $\S 5.1$ ), we tried both mechanisms: In simulation Q1.3-D, we drained orbital angular momentum from the system at a rate of $1 \%$ of $J_{\text {tot }}$ per orbit for two orbits; in simulation Q1.3-E, we forced a slow expansion of the donor by increasing $K_{d}$ at a rate of $1.67 \%$ of its initial value over a timescale of 1 orbit for the first two orbits (see Appendix $\mathrm{C}$ for details). A detailed comparison of simulations Q1.3-D and Q1.3-E confirms that the outcome of the instability is insensitive to the process by which the donor has been brought into contact with its critical Roche surface.

\subsection{Simulations with $M_{\mathrm{d}}=1.323 M_{\mathrm{a}}$ Initially}

\subsubsection{Evolution Q1.3-D}

Figure 3 presents a sequence of images showing the structure of the binary at selected times during the Q1.3-D evolution. Each image is a three-dimensional rendering of the mass-density distribution as viewed looking down on the equatorial plane of the binary from a frame of reference rotating with the initial orbital frequency, $\Omega_{0}$. The four nested iso-density surfaces have been drawn at levels $\rho / \rho_{a}^{\max }=0.5$ (green), 0.1 (yellow), $10^{-3}$ (red) and $10^{-5}$ (blue), where $\rho_{a}^{\max }$ is given in Table 4. The eight images presented in Figure 3 have been taken from an animation sequence that includes approximately 1500 frames (120 frames per orbital period) and illustrates in much more detail the dominant structures that developed during the Q1.3-D evolution. The first seven images displayed here are equally spaced in time at intervals of $2 P_{0}$. The gradual, counter-clockwise shift in the position angle of the line that connects the centers of the two stars reflects the gradual, 
monotonic increase in the system's orbital angular velocity $\Omega$, which in turn reflects a gradual decrease in the system's orbital separation, $a$.

By the end of the first two orbits, an accretion stream has begun to develop. We quickly appreciate that, for the selected mass-radius relationship of this binary system, the accretor has a sufficiently large radius that the mass-transfer stream must directly impact, rather than go into orbit around, the accretor. The material is moving supersonically as it hits the accretor's surface. This creates a standing shock wave of relatively high thermal-pressure material on the accretor's surface (visible as a ridge of material in the images shown at $t / P_{0}=6,8$, and 10) that balances the ram pressure of the in-falling donor material. The stream clearly strikes the surface of the accretor at an oblique angle. Hence, while the stream's motion perpendicular to the surface is abruptly halted by the standing shock front, its motion tangent to the surface continues unabated. In this manner, angular momentum is added to the spin of the accretor. It is precisely this complex, threedimensional flow - along with the tidal torques generated by the perturbed density distribution that characterizes direct-impact accretion and necessitates a fully three-dimensional hydrodynamic treatment to quantify its role.

Throughout the Q1.3-D evolution, the accretion stream steadily grows thicker, reflecting a steady increase in the rate at which material from the donor is being transferred to the accretor. By the time the system has completed twelve orbits, it can no longer be described as two stars in nearly circular orbits that are loosely tied together by a mass-transfer stream. Instead, the two stars have begun to plunge toward one another and, only one quarter of an orbit later (see Figure 3) the central cores of the two stars have merged. This final, short merger phase of the evolution results from what Lai et al. (1994a) have identified as a tidal instability. At late times a fairly steady stream of very low density material (identified by the blue iso-density surface) also emerges from the trailing edge of the less massive star, that is, the accretor, flows out through the $L_{2}$ Lagrange point, and accumulates along the boundary of the computational grid. In this manner, $\sim 0.1 \%$ of the total system mass is lost from the system during the first 12 orbits - a remarkably high level of conservation through the mass transfer event, given the very high mass-transfer rate attained prior to merger.

Table 4. Initial Parameters for Model Q1.3 ${ }^{\dagger}$

\begin{tabular}{lclcc}
\hline \hline $\begin{array}{c}\text { System } \\
\text { Parameter }\end{array}$ & $\begin{array}{c}\text { Initial } \\
\text { SCF Value }\end{array}$ & $\begin{array}{c}\text { Component } \\
\text { Parameter }\end{array}$ & Donor & Accretor \\
\hline$q_{0}$ & 1.323 & $M_{i}$ & 0.0176 & 0.0133 \\
$a_{0}$ & 0.8882 & $\rho_{i}^{\max }$ & 0.6000 & 1.0000 \\
$\Omega_{0}$ & 0.2113 & $K_{i}$ & 0.0372 & 0.0264 \\
$J_{\text {tot }}$ & $1.40 \times 10^{-3}$ & $V_{i}$ & 0.1810 & 0.0799 \\
$R_{\text {com }}$ & $7.11 \times 10^{-5}$ & $V_{i}^{\mathrm{RL}}$ & 0.1869 & 0.1261 \\
\hline
\end{tabular}

\footnotetext{
${ }^{\dagger}$ Parameter values are given here in dimensionless polytropic units. To scale these numbers to other (e.g., cgs) units, see the discussion in Appendix B.
} 
For purposes of further discussion it is productive to divide the Q1.3-D evolution into three phases that are contiguous in time: the "driving" phase $\left(0 \leq t / P_{0} \leq 2\right)$; the "mass-transfer" phase $\left(2 \leq t / P_{0} \leq 12\right)$; and the final "merger" phase $\left(12 \leq t / P_{0} \leq 12.25\right)$. More quantitative descriptions of the first two of these evolutionary phases are provided by Figures 4 and 5 . Figure 4 shows the time-dependent behavior of (top panel, solid line) the $z$-component of the system's total angular momentum, (middle panel) the system's orbital angular momentum, and (bottom panel) the spin angular momentum of both the donor (dot-dashed curve) and the accretor (dashed curve). In all three panels of Figure 4, the quantity being plotted is normalized to the system's initial total angular momentum, $J_{\text {tot }}$, and the curves have not been extended beyond $t / P_{0}=12$ because at later times it becomes difficult to distinguish between the individual stellar components. The solid curves in Figure 5 display the time-dependent behavior of the mass transfer rate $\dot{M}_{d}$ normalized to the ratio $\dot{M}_{\text {ref }} \equiv M_{d}(t=0) / P_{0}$ (top panel), the system mass ratio $q$ (middle panel), and the orbital separation $a$ normalized to $a_{0}$ (bottom panel). For reasons that will become apparent, below, in Figure 5 the time coordinate (horizontal axis) has been shifted to $t_{\text {merge }} \equiv\left(t_{\mathrm{D}}-12 P_{0}\right)$, where $t_{\mathrm{D}}$ is the evolutionary time recorded during the Q1.3-D simulation, so that the onset of the final merger phase occurs at the origin (i.e., at $t_{\text {merge }}=0$ ).

As was explained earlier, during the "driving" phase of the Q1.3-D evolution, angular momentum was artificially extracted from the system at a rate of $1 \%$ of $J_{\text {tot }}$ per orbit. This is directly reflected in the top panel of Figure 4, where the behavior of $J_{z}(t)$ can be compared with a (dotted) line whose slope is precisely $0.01 J_{\text {tot }} / P_{0}$. During this phase of the evolution, angular momentum was extracted from the system in such a way that the spin angular momenta of the two stellar components $J_{d}$ and $J_{a}$ (bottom panel of Figure 4) remained essentially unchanged. (See Appendix C for a precise description of this extraction method.) In practice, then, the extraction of angular momentum resulted in a steady drop in the system's orbital angular momentum over the first two orbits (see the middle panel of Figure 4) as, in a terminology that is consistent with the discussion associated with Eq. (11), the system experienced a systemic loss of angular momentum $\left(\dot{J} / J_{\text {orb }}\right)_{\text {sys }} \approx 0.01 / P_{0}$. According to Eq. (11), therefore, it is no surprise that during the driving phase of this evolution the orbital separation decreased at a rate of $\dot{a} / a_{0} \approx 0.02 / P_{0}$ (see the bottom panel of Figure 5 ), that is, to a value $a / a_{0} \approx 0.96$ after two orbits. This, in turn, produced an $\approx 4 \%$ reduction in the effective Roche-lobe radii, which produced the desired result of bringing the donor's Roche lobe into a sufficiently deep contact with the stellar surface of the donor that the mass-transfer event was initiated. As is shown in the top panel of Figure 5, at the end of this "driving" phase of the evolution (marked by the solid dot), $\left|\dot{M}_{d}\right| \approx 10^{-3} M_{d} / P_{0}$. This mass-transfer rate was sufficiently large (compared to $\dot{M}_{\text {min }}$, for example) that it produced a recognizable and spatially resolvable accretion stream (Figure 3).

At the end of the driving phase of the evolution, we stopped extracting angular momentum from the system; in effect, the external driving that had been producing a systemic loss of angular momentum $\left(\dot{J} / J_{\text {orb }}\right)_{\text {sys }}$ was set to zero. Throughout the remainder of the Q1.3-D evolution, therefore, the system's total angular momentum was conserved to a high level of precision (see the top panel of Figure 4, where the solid curve is perfectly horizontal), and variations in the orbital separation 
could only be attributed to the internal redistribution of angular momentum associated with mass transfer and tides, as is approximately described by the last two terms on the right-hand-side of Eq. (11).

A few key features are identifiable in the plot of $a(t)$ during the "mass-transfer" phase of the Q1.3-D evolution (solid curve in the bottom panel of Figure 5). First, the separation slowly, but steadily decreases in step with the slow, steady decrease of the system mass ratio $q$ (solid curve in the middle panel of Figure 5). This appears to be in accord with the explicit $q$-dependence of the last term on the right-hand-side of Eq. (11). Second, as was observed in the benchmark evolutions of model Q0.8, $a(t)$ displays a low-amplitude oscillation with a period $\approx P_{0}$. This "epicyclic" oscillation reflects the fact that the orbit is slightly noncircular. Although there is evidence that this epicyclic motion was amplified somewhat during the "driving" phase of this evolution, it is perhaps significant that the eccentricity of the orbit did not noticeably grow during the masstransfer phase. Third, in association with the onset of a tidal instability, $a(t)$ begins to decrease rapidly as $t_{\text {merge }}$ approaches zero.

As the solid curve in the top panel of Figure 5 shows, during the driving phase of the Q1.3-D evolution the accretion rate remains low as the surface of the donor is being brought closer to, and then into deeper contact with its Roche lobe. However, after the mass-transfer phase begins in ernest, the accretion rate steadily increases. This is as expected because, although the mass ratio $q$ steadily decreases throughout the mass-transfer phase of the evolution, it remains larger than unity until the rapid plunge and merger phase gets underway. (As shown in the middle panel of Figure 5, $q>1$ until $t / P_{0}=11.884$.) Hence, throughout most of this evolution, $R_{d}^{\mathrm{RL}}$ steadily decreases while $R_{d}$ steadily increases, so a larger and larger fraction of the donor's envelope rises above the critical Roche surface. This, in itself, is a formula for disaster and is sufficient to explain why merger was the inevitable outcome of this $q_{0}>1$ mass-transfer evolution. It should be noted as well that, as discussed in §2.2.3 above, the phenomenon of direct-impact accretion (Marsh \& Steeghs 2002; Marsh et al. 2004) must also have acted to further destabilize mass transfer in this system.

Throughout the mass-transfer phase of this evolution, $\dot{M}_{d}$ exhibits a low-amplitude oscillation on top of its steady, secular rise. This oscillation has a period $\approx P_{0}$, strongly suggesting that it is associated with the epicyclic oscillation seen in the plot of $a(t)$. This association is substantiated by the realization that, during each oscillation when $\dot{M}_{d}$ reaches a local maximum, the orbital separation $a$ is at a local minimum. That is, the simulation permits us to see variations in the mass-transfer rate that are directly associated with the slightly non-circular shape of the orbit. Because the eccentricity of the orbit (i.e., the amplitude of the epicyclic oscillations) does not noticeably increase during the evolution, whereas the mass-transfer rate does steadily increase, it is understandable that the relative amplitude of the oscillations that are seen in the plot of $\dot{M}_{d}(t)$ decrease with time. During the final two orbits of this evolution, the mass-transfer rate climbs rapidly to a very high amplitude, reaching a level $\gtrsim 10 \%\left(M_{d} / P_{0}\right)$ shortly before the final merger phase. This behavior of $\dot{M}_{d}(t)$ during the last portion of the mass-transfer phase of evolution Q1.3-D strongly resembles the behavior that has been predicted by the approximate analytical description of an unstable mass-transfer evolution presented by Webbink \& Iben (1987). 
The information plotted in Figure 4 allows us to examine, in part, the role that "direct impact" accretion plays in driving the evolution of this particular binary system. Throughout most of the mass-transfer phase of the evolution, the accretor gradually spins up (the dashed curve labelled $J_{a}$ in the bottom panel) as material from the stream is deposited onto its surface at an oblique impact angle. It is significant that the curves for $J_{a}(t)$ and $J_{\text {orb }}(t)$ are practically mirror images of one another, while the curve for $J_{d}(t)$ is almost perfectly flat until the final "plunge" occurs. This means that the spin-up of the accretor occurs almost entirely at the expense of the orbit. That is, at the time of impact, the specific angular momentum of the accretion stream material does not reflect the specific angular momentum of the star from which it originated (i.e., the donor) but instead reflects the amount by which the time-varying torque applied by the system's complex (and time-varying) gravitational field has been able to transfer angular momentum from the orbit to the stream as the stream material "falls" from the vicinity of the $L_{1}$ Lagrange point to the surface of the accretor. This result provides strong support for the arguments that have led Marsh et al. (2004) and Gokhale et al. (2005) to account for the effects of direct-impact accretion in their semi-analytical models of mass-transferring binary systems through a term that is built around the relatively simple concept of a circularization radius, as described above in the context of Eq. (11). At the very end of the "mass-transfer" phase of the Q1.3-D evolution, the binary loses orbital angular momentum to the spin of both stellar components catastrophically, as the stars plunge towards one another and eventually merge.

The above discussion suggests the following interpretation: initially and during most of the evolution, while the angular momentum of the donor remains flat, the system is evolving as a result of the mass transfer instability since $q>q_{\text {stable. }}$ Only near the end, tidal effects on the donor further reduce the orbital angular momentum causing the final merger. Thus this final phase should be considered the proper tidal instability. Binary systems in which direct impact occurs will have a harder time than disk systems avoiding the tidal instability since $q_{\text {stable }}<2 / 3$.

\subsubsection{Evolution Q1.3-E}

In an attempt to ascertain to what extent the outcome of a mass-transfer event depends on the manner in which the donor star is brought into contact with its critical Roche surface, we performed a second simulation in which the initial state was defined by model Q1.3 (with the associated parameters shown in Table 4) but, instead of artificially extracting angular momentum from the system, during the "driving" phase of the evolution we gradually increased the specific entropy of the gas inside the donor star. This caused a slow, secular increase in the effective radius of the donor and, within the first two orbits, brought the surface of the donor into sufficiently deep contact with its critical Roche surface that the mass-transfer event was initiated. More specifically, for this Q1.3-E evolution the polytropic constant $K_{d}$ for the material inside the donor was increased at a rate of $1.67 \%$ of its initial value per orbit for 2 orbits. (See Appendix C for a precise description of how this "driving" technique was implemented in the hydrocode.) Because $R \propto K$ in an $n=3 / 2$ polytropic star (see, for example, the discussion in $\S 2$ associated with Eq. 3), this level of driving 
should have caused the effective radius of the donor to increase by approximately $3.3 \%$ by the end of the driving phase of the Q1.3-E evolution.

As was anticipated, the results of this Q1.3-E simulation were very similar to the results of the Q1.3-D simulation. The accretion stream that had become well-defined by the end of the "driving" phase grew steadily thicker throughout the "mass-transfer" phase and, shortly after the system mass-ratio dropped below unity, the cores of the two stars catastrophically merged into a single object. There were, however, quantifiable differences between the two model evolutions. Most noticeably, the stars in the Q1.3-E evolution merged at an earlier evolutionary time. We expected that the two evolutions would be somewhat offset in time from one another. Given that two distinctly different mechanisms were employed to drive the system into contact, it was unlikely that, for example, $\dot{M}_{d}$ would be precisely the same in both simulations at the end of the driving phase (beginning of the mass-transfer phase) hence, it was unlikely that a basic system parameter such as $q$ would be precisely the same at a given time, $t$, because its value depends on the integral over $\dot{M}_{d}$ up to the time $t$. Before making further comparisons between these two simulations, we decided to synchronize their evolutionary times at the end, rather than at the beginning, of the mass-transfer phase. More specifically, we took a plot of $\log \left|\dot{M}_{d}\right|$ versus time from the Q1.3-D simulation (solid curve in the top panel of Figure 5) and slid it horizontally across the analogous plot from the Q1.3-E simulation - normalized in the same manner as the top panel of Figure 5 - until a reasonable match between the two time-dependent functions was achieved at late times. In this manner we were able to identify a "merger time" for the Q1.3-E evolution that could be reasonably well associated with the merger time $t_{\text {merge }}$ that was defined earlier for the Q1.3-D evolution. For the Q1.3-E evolution, a time offset of 5.9P $P_{0}$ was required to align the origin in time with the end of the mass-transfer phase, that is, $t_{\text {merge }} \equiv\left(t_{E}-5.9 P_{0}\right)$, where $t_{E}$ is the evolutionary time recorded during the Q1.3-E simulation. By comparison, a time offset of $12.0 P_{0}$ was required to arrange the same alignment for the Q1.3-D evolution.

The dotted curves in the top, middle, and bottom panels of Figure 5 display the behavior of, respectively, $\log \left(\left|\dot{M}_{d}\right| / \dot{M}_{\text {ref }}\right), q$, and $a / a_{0}$ as a function of $t_{\text {merge }} / P_{0}$ from simulation Q1.3-E. The mass-transfer rate and the function $q\left(t_{\text {merge }}\right)$ for this simulation match the mass-transfer rate and the function $q\left(t_{\text {merge }}\right)$ for simulation Q1.3-D very well over the entire "mass-transfer" phase of its evolution. The dotted curve for $\dot{M}_{d}\left(t_{\text {merge }}\right)$ exhibits lower-amplitude oscillations than the corresponding solid curve, indicating that the binary orbit has remained more nearly circular throughout the Q1.3-E evolution. In the plot of the time-dependent behavior of the binary separation (bottom panel of Figure 5), the solid curve lies below the dotted curve at all times. This is understandable because, due to the manner in which the system was initially driven into contact, the model in simulation Q1.3-D (solid curve) has a slightly smaller orbital angular momentum than the model in simulation Q1.3-E (dotted curve) at all times.

The top panel of Figure 5 explicitly shows that, at the end of the driving phase of simulation Q1.3-E $\left(t_{E}=2 P_{0}\right.$, hence $\left.t_{\text {merge }}=-3.9 P_{0}\right)$, the mass-transfer rate was higher than it was at the end of the driving phase of simulation Q1.3-D $\left(t_{D}=2 P_{0}\right.$, hence $\left.t_{\text {merge }}=-10 P_{0}\right)$. As was forecast, above, this in itself explains why the Q1.3-E simulation merged more quickly. Had we stopped 
"driving" the expansion of the donor somewhat sooner - say, after only 1.5 orbits instead of after 2 orbits $-\dot{M}_{d}$ would have been smaller at the onset of the mass-transfer phase of simulation Q1.3-E and it would have taken longer for the two stars to merge. Nevertheless, the mass-transfer phases of the two separate Q1.3 model simulations are remarkably similar and certainly the outcome of the evolutions (catastrophic merger) is the same. This supports our expectation that the endpoint of a mass-transfer evolution will be insensitive to the manner in which the binary system is initially brought into contact.

The top half of Figure 6 contains six frames from the animation sequence (mentioned earlier) that was generated to visually illustrate the complex fluid-dynamical flows that developed during simulation Q1.3-D. Four of these images are identical to ones that were presented earlier in Figure 3 , but here the evolutionary time $t_{\text {merge }}$ has been used to identify when each image was drawn from the simulation. For comparison, images from simulation Q1.3-E are displayed in the bottom half of Figure 6 at the same synchronized times, $t_{\text {merge }}$. Each of the last two images in the bottom row of Figure 6 is remarkably similar to its corresponding image in the top row of the Figure, given that they represent highly distorted structures that have developed from complex, nonlinear, mass-transfer evolutions and given that they have arisen at two quite different "absolute" times in the separate numerical simulations. (At earlier times, e.g., $t_{\text {merge }}=-1 P_{0}$, the binary structures are also quite similar, although there is an identifiable orbital phase discrepancy due to the fact that the orbital separations and, hence, the orbital frequencies are slightly different in the two simulations.) The correspondence between these highly distorted structures at late times in these two separate simulations provides perhaps the strongest empirical evidence that the endpoint of a mass-transfer evolution will be insensitive to the manner in which a binary system is initially brought into contact. At the same time, the quantitative similarity between the late-time results of evolutions Q1.3-D and Q1.3-E serves as a strong, affirmative convergence test for our hydrocode.

\subsection{Simulations with $M_{\mathrm{d}}=0.5 M_{\mathrm{a}}$ Initially}

As an example of mass transfer in binaries with $q<2 / 3$, we now discuss the results of three Q0.5D simulations in which driving was applied for different times and compare the results with those obtained by RS95 for a DWD binary of identical mass ratio (hereafter, referred to as simulation Q0.5-RS). Building on our detailed analysis and description of the model Q1.3 evolutions, the Q0.5-D simulations can be described fairly concisely. In particular, Figures 7-10 display selected results from our Q0.5-D evolutions in the same manner in which results from simulation Q1.3-D were presented in Figures 3-5.

Initially, the donor star in our model Q0.5-D simulations was slightly detached from its Roche lobe: $V_{\mathrm{d}} / V_{\mathrm{d}}^{\mathrm{RL}}=0.90$ (Table 5). In the first of our three Q0.5-D runs, henceforth simulation Q0.5-Da, in order to bring the system into contact and initiate the mass-transfer event, angular momentum was drained from the binary - the "driving" phase of the simulation - at a rate of $1 \%$ per orbital period over 2.7 periods. The system was then allowed to evolve in the absence of driving - the "mass-transfer" phase of the evolution - since there was no driving present in the 
simulations of RS95 and because our primary objective was to see if mass transfer, once initiated, is unstable. In a second Q0.5-D simulation, subsequently referred to as Q0.5-Db, we extended the driving phase to 5.3 orbits at the same level of $1 \%$ per orbit, and then let it evolve without driving. This allowed us to investigate the effects of deeper initial contact and a higher mass-transfer rate on the evolution of the binary. Finally, in a third simulation, referred to as Q0.5-Dc, we applied driving at the rate of $1 \%$ per orbit throughout the evolution. The length of time over which angular momentum was extracted from the system in all three of these Q0.5-D simulations is reflected in panel (a) of Figure 8. During the driving phase the angular momentum follows closely the dotted line, turning horizontal when the driving stops for evolutions Q0.5-Da (blue curve) and Q0.5-Db (green curve). The Q0.5-Dc evolution (red curve) is driven throughout, so it follows the dotted line to the end. According to expectations, the longer we applied driving, the shorter the time it took for the binary to evolve to a comparable mass transfer level and evolutionary stage. Consequently, the longest of these three simulations was Q0.5-Da (32 orbits), while Q0.5-Dc was the shortest (8.5 orbits).

We focus first on Figure 7, which displays images of the mass-density distribution at selected time slices during the Q0.5-Da evolution, and on the blue curve in the bottom panel of Figure 9, which shows the time-evolutionary behavior of the binary separation for this same model evolution. During the brief "driving" phase of the evolution, the separation decreases at a rate that is consistent with the rate at which angular momentum is being extracted from the system. An epicyclic oscillation with a period $\approx P_{0}$ and an amplitude of just over $1 \%$ is present in the $a(t)$ curve; as before, we suspect this arises from initial conditions and driving. In addition there is another oscillation with a period of about $3 P_{0}$ which appears to be related to a slow meandering of the position of the center of mass of the system (see the discussion, below, related to Figure 11). We note that these oscillations also appear - although at a somewhat reduced amplitude - in evolutions Q0.5-Db (green curve) and Q0.5Dc (red curve), and they are mirrored in the mass transfer rates shown in the top panel of Figure 9.

After driving has been turned off in evolution Q0.5-Da, the separation hovers around a value $a \approx 0.95 a_{0}$ for more than twenty orbits, then the binary begins to separate and the mass-transfer

Table 5. Initial Parameters for Model Q0.5 ${ }^{\dagger}$

\begin{tabular}{lclcc}
\hline \hline $\begin{array}{c}\text { System } \\
\text { Parameter }\end{array}$ & $\begin{array}{c}\text { Initial } \\
\text { SCF Value }\end{array}$ & $\begin{array}{l}\text { Component } \\
\text { Parameter }\end{array}$ & Donor & Accretor \\
\hline$q_{0}$ & 0.500 & $M_{i}$ & $3.073 \times 10^{-3}$ & $6.143 \times 10^{-3}$ \\
$a_{0}$ & 0.8764 & $\rho_{i}^{\max }$ & 0.235 & 1.0000 \\
$\Omega_{0}$ & 0.1174 & $K_{i}$ & 0.016 & 0.016 \\
$J_{\text {tot }}$ & $1.97 \times 10^{-4}$ & $V_{i}$ & 0.0814 & 0.0370 \\
$R_{\text {com }}$ & $1.04 \times 10^{-5}$ & $V_{i}^{\mathrm{RL}}$ & 0.0906 & 0.2380 \\
\hline
\end{tabular}

${ }^{\dagger}$ Parameter values are given here in dimensionless polytropic units. To scale these numbers to other (e.g., cgs) units, see the discussion in Appendix B. 
rate (blue curve in the top panel of Figure 9) levels off. The images in Figure 7 reflect this $a(t)$ behavior. Initially the binary advances in the corotating frame as its orbital frequency increases. At late times, as the separation increases, the binary slows down, stalls and after some hesitation due to the various oscillations described above, it moves in a retrograde sense when the orbital frequency falls below $\Omega_{0}$ at $t \approx 31 P_{0}$. (This behavior is quite clear in the $\approx 3800$-frame animation sequence from which the individual images shown here were extracted.) In summary, we have followed the Q0.5-Da evolution through more than 30 orbits in the presence of a steady mass-transfer stream and there is no indication that the system is going to merge. This result is significantly different from the Q0.5-RS evolution, which was violently unstable to mass transfer and led to tidal disruption of the donor within $\sim 5$ orbits.

In an effort to understand why our Q0.5-Da evolution differed from the Q0.5-RS evolution, we extended the initial "driving" phase to 5.3 orbits (model Q0.5-Db) in order to bring the Roche lobe into deeper contact with the donor and bring the system to a higher mass-transfer rate as it entered the "mass-transfer" phase of its evolution. As the green curve in the bottom panel of Figure 9 shows, the orbital separation steadily decreased (as expected) during the extended "driving" phase of this evolutions, but after the driving ceased the separation $a(t)$ evolved in a slightly different manner from the Q0.5-Da evolution. Specifically, the system did not hover at its minimum separation as long; it began separating and its mass-transfer rate began to level off after only $\approx 11 P_{0}$. We can understand this difference in behaviors if we refer to Eq. (11).

In the absence of tidal effects, Eq. (11) predicts that the orbital separation should increase as soon as driving is interrupted. [Note that this result holds even when there is a significant consequential transfer of orbital angular momentum to the spin of the accretor - see panels (b) and (c) of Figure 8 where, as was seen in the Q1.3-D evolution, $J_{\mathrm{a}}(t)$ is practically a mirror image of $J_{\text {orb }}(t)$.] However, after the driving phase has ended in evolution Q0.5-Da, the separation does not immediately begin to increase; in fact, a careful examination of the blue curve in the bottom panel of Figure 9 shows that the separation continues to decrease, albeit at a much reduced rate. This deviation from the "expected" behavior must be attributed to the tidal terms in Eq. (11). The blue curve in panel (d) of Figure 8 provides evidence that tides are at work: over the first approximately 14 orbits of evolution Q0.5-Da, the donor is also being spun up, storing part of the orbital angular momentum and thereby allowing the separation to continue to decrease slowly. This is readily understandable because the initial driving rapidly shrinks the binary, thereby increasing the orbital frequency. The spin of the donor lags initially and recovers gradually as it is spun up by tides. Some time after $J_{\mathrm{d}}$ peaks at $t \sim 14 P_{0}$, the binary separation finally begins to increase at $t \sim 18-20 P_{0}$, and the masstransfer rate levels off. In evolution Q0.5-Db, the separation stops decreasing almost immediately after the driving phase has ended (see the green curve in Figure 9). It increases slowly at first, then much more rapidly at the end of the simulation. Because the driving phase lasted longer in this evolution than in Q0.5-Da, the mass-transfer rate was able to climb to a sufficiently high level by the time driving was stopped to permit the " $\dot{M}_{d}$ " term in Eq. (11) to exceed the negative tidal terms.

At the end of both the Q0.5- $\mathrm{Da}$ and Q0.5-Db simulations, not only is the binary separation 
increasing, but the mass-transfer rate has leveled off. We speculate that if we were able to follow these evolutions significantly farther in time in the absence of driving, the magnitude of $\dot{M}_{d}$ would steadily decrease and we would find that the binary eventually detaches and mass-transfer ceases. We also conjecture that the mass of the remnant donor would be a decreasing function of the level and duration of the original phase of driving. Confirmation of these conjectures must await further improvements in our simulation tools (see further discussion, below).

In order to investigate how an even higher mass-transfer rate might affect the evolution of this $q_{0}=0.5$ binary system, we performed a third simulation (model Q0.5-Dc) with continuous "driving" at a rate of $1 \%$ per orbit. Because driving was never turned off, systemic angular momentum losses played a dominant role in dictating how the orbital parameters of the system varied throughout most of this evolution. As the red curve in the bottom panel of Figure 9 shows, the orbital separation steadily decreased for $\approx 8 P_{0}$ at a rate that would be predicted by the first term alone on the righthand-side of Eq. (11). As a consequence, the Roche lobe sank quite deep into the envelope of the donor and, as depicted by the red curve in the top panel of Figure 9, the mass-transfer rate steadily grew throughout the evolution, reaching a level that was an order of magnitude higher than the maximum rate acquired in evolution Q0.5- $\mathrm{Db}$ and roughly two orders of magnitude higher than the maximum rate acquired in evolution Q0.5-Da. Even under these extreme conditions, however, the orbital separation eventually reached a minimum (at $t / P_{0} \approx 8$ ) and the system began separating (red curve in the bottom panel of Figure 9). Presumably this reversal occurred because the masstransfer rate became large enough for the " $\dot{M}_{d}$ " term in Eq. (11) to finally dominate over systemic angular momentum losses.

As the images in Figure 10 illustrate, through approximately $7 P_{0}$, model Q0.5-Dc evolved through configurations that resemble those seen in the Q0.5-Da evolution (see Figure 7). But late in the evolution, the accreted material has formed a much more prominent, time-dependent, nonaxisymmetric disk-like structure around the accretor; and at the end of the simulation, the donor is being tidally ripped apart, even as the measured orbital separation is increasing. This evolution was terminated when a significant fraction of material from the tidally elongated donor hit the outer edge of the computational domain. Interestingly, the evolution of this "driven" system during its final 2-3 orbits bears a strong resemblance to the final 2-3 orbits of the published Q0.5-RS evolution.

For evolutions Q0.5- $\mathrm{Da}$ and Q0.5-Db, we think that our results are more aptly described as the donor being gradually stripped of its mass and partially disrupted by tides rather than as a catastrophic merger of the binary. We suspect that, if these evolutions could be followed accurately beyond what the present version of our code is able to do, a sizeable portion of the donor would survive (final mass ratio $q \sim 0.3$ ) in an elliptical orbit. In fact, even in evolution Q0.5-Dc it appears that although the donor is largely tidally disrupted, a small remnant may survive at a larger radius. Unfortunately, because of the growing drift of the center of mass (see Figure 11), we cannot follow evolutions Q0.5-Da and Q0.5-Db far enough to unambiguously show that a long-lived remnant of the donor survives the tidal disruption. Despite these limitations, our results are significantly different from the Q0.5-RS evolution, which was violently unstable to mass transfer and led to tidal disruption of the donor within $\sim 5$ orbits. As was briefly forecast in $\S 2.2$, and as has been discussed 
in early paragraphs of this section of the paper, the results we have obtained are consistent with the behavior expected from Eq. (11). They also are consistent with the results of integrations of orbit-averaged evolution equations to be described elsewhere (Gokhale et al. 2005).

A possible reason that the Q0.5-RS binary was found by RS95 to be unstable to mass transfer is that the donor may have been in deeper contact with its critical Roche surface at the start of their simulation. To test this we have calculated the values of the separation $r$ (as defined in RS95) at the point the driving is terminated in our simulations and have compared it with RS95. In case Q0.5-Da, $r \approx 4.0$, which is greater than the initial separation, $r=3.9$, reported by RS95 for evolution Q0.5-RS (note that in RS95 the donor is in contact with its Roche lobe at the outset of the simulation). It appears as though the two stars are initially closer to each other in simulation Q0.5RS and, hence, the donor is in deeper contact with its critical Roche surface initially. In turn, this implies that the mass-transfer simulation conducted by RS95 started with a higher accretion rate. From Figure 13a of RS95, $\log \left|\dot{M}_{\mathrm{d}}\right| \approx-1$ after only 4 orbits, which is higher than the mass transfer rate we observed at any stage of our Q0.5-Da simulation. In case Q0.5-Db, $r \approx 3.8$, very close to the initial conditions of RS95, and yet the accretion rate appears to peak below $\log \left|\dot{M}_{\mathrm{d}}\right| \approx-1$. Finally, in run Q0.5-Dc, the separation appears to turn around at the end when $r \approx 3.6$, above the value at which we expect the tidal instability to set in. Unfortunately, because the donor bumps up against the edge of our computational domain at the end of our simulation, we cannot follow model Q0.5-Dc far enough to deduce its ultimate fate. However, the accretion rate is still growing rapidly while the orbital angular momentum is plummeting suggesting a full tidal disruption and perhaps eventual merger. Determining whether the donor survives in this case remains an obvious goal for future simulations.

We also note that in the Q0.5-RS evolution, the distance between the centers of mass of the two stars decreases while the density maxima of the two components separate. This behavior seems odd, but can perhaps be understood if the mass in the stream is large enough to significantly shift the center of mass of the distorted donor in the downstream direction. In our Q0.5-D simulations the mass in the stream is always a small fraction of the donor's mass. As a result, both the distance between the centers of mass of the binary components and the separation of their density maxima increase at late times thus saving the donor from tidal disruption, except perhaps in the Q0.5-Dc case.

It should be emphasized that, in our Q0.5-D simulations, the adopted initial driving rate was orders of magnitude larger than what one would expect in a realistic DWD binary. A milder driving favors stability since all effects discussed above will also be milder. With driving applied throughout the entire Q0.5-Dc evolution at the initial rate of $1 \%$ per orbit, a tidal disruption may well be the final outcome, and it remains to be seen if any fraction of the donor survives. The behavior observed in this case comes closest qualitatively to the evolution reported by RS95 for the Q0.5-RS simulation.

Finally, Figure 11 shows the time-dependent meandering of the position of the center of mass of the Q0.5 binary system throughout our Q0.5-D simulations. Its radial distance from the cylindrical coordinate axis, $R_{\text {com }}$ (bottom panel), and its associated equatorial-plane Cartesian coordinates 
( $x_{\text {com }}$, top panel; and $y_{\text {com }}$, middle panel) as viewed from a frame of reference rotating with the initial orbital frequency, $\Omega_{0}$, are plotted as a function of $t / P_{0}$. As was described by MTF and discussed in $\S 3.1 .2$ above, there is a tendency for an unequal-mass binary system to drift away from the cylindrical coordinate axis during a simulation. If left unchecked, some part of the binary would hit the boundary of the grid in a time $\sim 10 P_{0}$. During our Q0.5-D simulations the corrections described in $\S 3.1 .2$ have succeeded in confining the center of mass to within $\sim 1$ zone of the computational grid up to the time when the binary begins to separate rapidly and the donor approaches the boundary. In run Q0.5-Da the simulation remains well-behaved for over 30 orbits. This is significantly longer than any other self-consistent hydrodynamic simulation of a binary evolution with or without mass-transfer that we are aware of.

Despite this success, however, there is still room for improvement. For example, it appears that the residual center-of-mass motion shown in Figure 11 has been reflected in an undesirable way in other dynamical features of simulation Q0.5-D. Most noticeably, the oscillation that is seen in all three panels of Figure 11 with a period $\sim 3 P_{0}$ and an amplitude $\sim \Delta R / a_{0}=0.9 \%$ appears to be modulating the natural epicyclic oscillations that appear in the functions $a(t)$ and $\dot{M}_{\mathrm{d}}(t)$ throughout the mass-transfer phase of the evolution (see the bottom and top panels of Figure 9). In addition, we have observed that, with the correction in place, as the binary begins to separate rapidly, a numerical instability can eventually cause the center of mass to rapidly spiral outward. We have stopped the Q0.5-D simulations before this instability sets in.

\section{Discussion and Conclusions}

This is the second in a series of papers that describe results of direct simulations of the dynamical evolution of unequal-mass binaries using a three-dimensional, finite-difference hydrodynamics technique. Our long-term goal is to gain a better understanding of the origin and survival of various classes of binaries - including double white dwarfs, contact binaries and direct-impact accretors

- by accurately simulating hydrodynamical flows that arise when binaries of various kinds become semi-detached. In contrast to this, much of the related analytic and numerical work that has been carried out over the past decade has placed an emphasis on the onset of the tidal instability and on the coalescence and merger of nearly identical neutron stars. In these earlier studies, mass transfer events were modeled when necessary as a prelude to the almost inevitable merger. In fact, the inevitability of mergers seems to have been accepted also for double white dwarf binaries. While this may well be the case with massive white dwarfs, the results presented here already suggest that low-mass WD binaries may escape merger and survive as AM CVn systems (Tutukov \& Yungelson 1996; Nelemans, et al. 2001).

In the present paper, after describing improvements that have been made in the hydrodynamics code that was developed by and described in MTF, we have taken the first steps toward elucidating the role that is played by mass transfer in the outcome of the dynamical phase of evolution following first contact. We have been especially concerned with those binary systems in which the mass ratios and equations of state are such that Roche lobe contact occurs before a tidal instability, so we have 
focused on binaries with $n=3 / 2(\gamma=5 / 3)$ polytropic components (Rasio \& Shapiro 1995; Uryu \& Eriguchi 1998a,b). We have presented two evolutions of a system having an initial mass ratio $q_{0}=1.323$ that was dynamically unstable to mass transfer. In one evolution, mass transfer was initiated by removing angular momentum from the binary (Q1.3-D) while in the other evolution we expanded the donor star causing it to overflow its Roche lobe (Q1.3-E). While there were subtle differences in the structure of the binary and the depth of contact by the time driving was terminated, in both cases the binary was dynamically unstable and a merger was the final result. In fact, we demonstrated that if one looks at the last two or three orbital periods before the merger, the behavior of both evolutions is consistent with being the same. We therefore conclude that, for unstable systems, it does not matter how the system gets into contact; once the instability kicks in, the behavior is the same. This result also has served as a convergence test for our hydrodynamics code since two evolutions with identical initial states but that were brought into contact in different ways and therefore had different detailed histories, ended up looking indistinguishable during the final stages leading to a merger.

At the very end of the "mass-transfer" phase of both the Q1.3-D and Q1.3-E evolutions, the binary transferred orbital angular momentum to the spin of both components catastrophically, as the stars plunged towards one another and eventually merged. Overall the behavior we observed during the final few orbits agrees with the predictions of the tidal instability (Lai et al. 1993a,b, 1994a,b,c). This suggests that one can recognize two epochs during the evolution of a $q_{0}>q_{\text {stable }}$ binary system that undergoes an episode of mass-transfer: Over an extended period of time, the rate of mass transfer grows while the orbital angular momentum changes very slowly. This instability is driven by an ever increasing depth of contact and thus can be considered a mass-transfer instability. Once the separation has been reduced sufficiently, the second instability sets in, during which the orbital angular momentum drops rapidly while the spin angular momenta increase driven by tides.

We have also presented detailed results from three mass-transfer evolutions (simulations Q0.5$\mathrm{Da}, \mathrm{Db}$, and $\mathrm{Dc}$ ) of a polytropic binary with an initial mass ratio $q_{0}=0.5$ and in which the two components had the same specific entropy. Thus this initial state could represent a DWD system with components having the same composition. It also corresponds to one system that was simulated by Rasio \& Shapiro (1995) using an SPH technique. In each of our simulations, the less massive star was driven into contact with its Roche lobe via the slow removal of orbital angular momentum, but in simulations Q0.5-Da and Q0.5-Db this artificial driving was turned off shortly after the mass-transfer event was initiated (after 2.7 and 5.3 initial orbital periods, respectively). Our results differ in some important ways from the results reported by RS95. Most significantly, in the two simulations that were evolved for an extended period of time in the absence of systemic angular momentum losses, we do not observe a merger nor a tidal disruption; in evolution Q0.5-Da (Db) our binary survives for more than 30 (14) orbital periods and at the end it is separating while the mass-transfer rate has leveled off. Via these extended simulations, we have demonstrated that our numerical tools permit us to accurately model accretion flows in dynamically evolving masstransfer systems. We can, for example, analyze how angular momentum is exchanged between the orbit and the spin of the two stars during a phase of direct-impact accretion, and we can 
analyze how the structure of the accretor dynamically readjusts as relatively high specific angular momentum material is deposited onto its equatorial region. Studies of this type should assist in the determination of which simplifying assumptions are justified - as well as which are not - in models that attempt to describe extended phases of mass-transfer evolutions semi-analytically (Webbink \& Iben 1987; Marsh et al. 2004). By conducting a variety of related simulations, we ultimately hope to be able to determine what the critical mass ratio $q_{\text {stable }}$ is that defines which binary systems are stable or unstable against mass-transfer.

We speculate that the outcomes of our Q0.5- $\mathrm{Da}$ and Q0.5-Db simulations were different from the Q0.5-RS simulation because the evolution presented in Rasio \& Shapiro (1995) was started from a significantly deeper initial contact, and thus transferred a larger fraction of the mass before separating. In RS95, the maximum density of the donor appears to be moving away and yet the separation calculated as the distance between the centers of mass of the material in their respective Roche lobes seems to be decreasing. Therefore, our results could be described as reproducing some of the initial features of the SPH simulation by Rasio \& Shapiro (1995) at a slower pace. Evidently, if the mass ratio of the binary is such that initially the system is unstable to mass transfer, the final fate of the binary will depend on whether during the initial phase of mass transfer the separation decreases sufficiently for the tidal instability to take over. In our Q0.5-Dc simulation, in which driving remained on throughout the evolution, it appears as though the system encounters the tidal instability; the last 2-3 orbits of this evolution resemble fairly closely the evolutionary behavior of the published Q0.5-RS simulation. However, in our Q0.5- Da and Q0.5-Db simulations, once the driving is cut off the tidal effects only succeed in delaying the tendency of the binary to separate as mass transfer proceeds by temporarily storing some orbital angular momentum in the spin of the donor. Once the separation begins to increase, tides become ineffective and the binary avoids the merger.

Given an equation of state for the binary components, the mode of mass transfer, and the expected mass loss, if any, it is possible to make predictions about the evolutionary outcome after contact. However, our simulations suggest that the eventual fate of such a binary depends not only on the

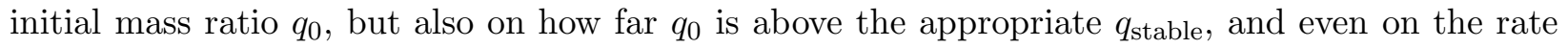
of driving unless this is very slow. In other words, the detailed outcome depends on the non-linear development of the mass-transfer and tidal instabilities. Only when $q_{0}$ is well above $q_{\text {stable }}$ will the evolution of mass transfer proceed rapidly enough to resemble qualitatively the predictions of the analytic solution derived in Webbink \& Iben (1987). When $q_{0}$ is only slightly above $q_{\text {stable }}$, non-linear effects come into play that make it possible for the system to survive the mass-transfer instability and avoid merger. We shall discuss these questions further in two forthcoming papers (Gokhale et al. 2005; Motl et al. 2005). As has already been mentioned, the tools we have developed will also enable us in the future to investigate in more detail the hydrodynamics of mass transfer and the structures arising from this transfer, transient flows, oscillations, mixing and convection. We see already some of these features in our simulations and we think they warrant further investigation.

This work has been supported in part by NSF grants AST 04-07070 and PHY 03-26311, and in part through NASA's ATP program grants NAG5-8497 and NAG5-13430. The computations 
were performed primarily at NCSA through grant MCA98N043, which allocated resources on the Tungsten cluster, and on the SuperMike and SuperHelix clusters at LSU, which are operated by the Center for Computation and Technology (CCT). J. E. T. acknowledges support from the NSFsponsored Institute for Pure and Applied Mathematics at UCLA, which provided an environment in May, 2005 that was conducive to writing significant portions of this manuscript. We thank the referee for many insightful comments and for encouraging us to perform some additional simulations.

\section{A. Correction for Center-of-Mass Motion}

In $§ 3.1 .2$, we presented an analytic expression for a small "artificial" acceleration $\mathbf{a}^{\text {art }}$ that has been added to the source term of the equation of motion in an effort to counteract a slow wandering of the center-of-mass of the binary system in each of our simulations. Here we describe how the various terms in expression (14) have been evaluated in the hydrocode.

In practice, at each discrete point in time $t^{(n)}$, the location $\mathbf{r}_{\mathrm{com}}^{(n)}=\left[\mathbf{i} x_{\mathrm{com}}^{(n)}+\mathbf{j} y_{\mathrm{com}}^{(n)}+\mathbf{k} z_{\mathrm{com}}^{(n)}\right]$ of the center of mass of the system has been determined numerically via an appropriate integral over the mass-density distribution, $\rho^{(n)}$. Then, for example, knowing the $x$-component of the center-of-mass position from the present and two previous points in time, $t^{(n-1)}$ and $t^{(n-2)}$, the $x$-components of the center-of-mass velocity and acceleration have been determined empirically through the respective finite-difference expressions,

$$
\begin{aligned}
& \dot{x}_{\mathrm{com}}^{(n)}=\frac{x_{\mathrm{com}}^{(n)}\left\{2 \Delta t^{(n-1)} \Delta t^{(n-2)}+\left[\Delta t^{(n-2)}\right]^{2}\right\}-x_{\mathrm{com}}^{(n-1)}\left[\Delta t^{(n-1)}+\Delta t^{(n-2)}\right]^{2}+x_{\mathrm{com}}^{(n-2)}\left[\Delta t^{(n-1)}\right]^{2}}{\Delta t^{(n-1)} \Delta t^{(n-2)}\left[\Delta t^{(n-1)}+\Delta t^{(n-2)}\right]} \\
& \ddot{x}_{\mathrm{com}}^{(n)}=2\left\{\frac{x_{\mathrm{com}}^{(n)}\left[\Delta t^{(n-2)}\right]-x_{\mathrm{com}}^{(n-1)}\left[\Delta t^{(n-1)}+\Delta t^{(n-2)}\right]+x_{\mathrm{com}}^{(n-2)}\left[\Delta t^{(n-1)}\right]}{\Delta t^{(n-1)} \Delta t^{(n-2)}\left[\Delta t^{(n-1)}+\Delta t^{(n-2)}\right]}\right\}
\end{aligned}
$$

where $\Delta t^{(n-1)} \equiv\left[t^{(n)}-t^{(n-1)}\right]$ and $\Delta t^{(n-2)} \equiv\left[t^{(n-1)}-t^{(n-2)}\right]$. Both of these expressions were derived from a straightforward Taylor-series expansion between the discrete coordinate positions at various times; analogous expressions produced measured values of the other cartesian components of the center-of-mass velocity and acceleration, $\dot{y}_{\mathrm{com}}^{(n)}, \dot{z}_{\mathrm{com}}^{(n)}, \ddot{y}_{\mathrm{com}}^{(n)}$, and $\ddot{z}_{\mathrm{com}}^{(n)}$. Finally, expressed in terms of the cylindrical coordinates used in the hydrocode,

$$
\begin{array}{rll}
a_{R, \mathrm{com}}^{(n)}=\ddot{x}_{\mathrm{com}}^{(n)} \cos \phi+\ddot{y}_{\mathrm{com}}^{(n)} \sin \phi & ; & v_{R, \mathrm{com}}^{(n)}=\dot{x}_{\mathrm{com}}^{(n)} \cos \phi+\dot{y}_{\mathrm{com}}^{(n)} \sin \phi \\
a_{\phi, \mathrm{com}}^{(n)}=\ddot{y}_{\mathrm{com}}^{(n)} \cos \phi-\ddot{x}_{\mathrm{com}}^{(n)} \sin \phi & ; & v_{\phi, \mathrm{com}}^{(n)}=\dot{y}_{\mathrm{com}}^{(n)} \cos \phi-\dot{x}_{\mathrm{com}}^{(n)} \sin \phi \\
a_{z, \mathrm{com}}^{(n)}=\ddot{z}_{\mathrm{com}}^{(n)} & ; & R_{\mathrm{com}}^{(n)}=\left\{\left[x_{\mathrm{com}}^{(n)}\right]^{2}+\left[y_{\mathrm{com}}^{(n)}\right]^{2}\right\}^{1 / 2}
\end{array}
$$

\section{B. Conversion of Code Units to Physical Units}

All of the initial model parameter values in Tables 1,4 , and 5 are given in units such that $G=R_{\mathrm{SCF}}=\rho_{\mathrm{a}}^{\max }(t=0)=1$, where $R_{\mathrm{SCF}}$ is the outer edge of the cylindrical grid that was used to generate the model in the SCF code. Fairly straightforward relationships can be used to scale the values of these dimensionless parameters to more meaningful (e.g., cgs) units. For example, if 
the total mass and orbital period of the Q0.8 binary configuration are specified in cgs units as $M_{\text {cgs }}$ and $P_{\text {cgs }}$, respectively, then the orbital separation of the system in centimeters is,

$$
a_{\mathrm{cgs}}=\left[\frac{\Omega_{0}^{2} a_{0}^{3}}{M_{\mathrm{a}}+M_{\mathrm{d}}}\right]^{1 / 3}\left[\frac{G M P^{2}}{4 \pi^{2}}\right]_{\mathrm{cgs}}^{1 / 3}=1.015\left[\frac{G M P^{2}}{4 \pi^{2}}\right]_{\mathrm{cgs}}^{1 / 3}
$$

the effective radius of the accretor in centimeters is,

$$
R_{a}^{\mathrm{cgs}}=\frac{a_{\mathrm{cgs}}}{a_{0}}\left(\frac{3 V_{\mathrm{a}}}{4 \pi}\right)^{1 / 3}=\left[\frac{3 V_{a} \Omega_{0}^{2}}{4 \pi\left(M_{a}+M_{d}\right)}\right]^{1 / 3}\left[\frac{G M P^{2}}{4 \pi^{2}}\right]_{\mathrm{cgs}}^{1 / 3}=0.351\left[\frac{G M P^{2}}{4 \pi^{2}}\right]_{\mathrm{cgs}}^{1 / 3},
$$

and the maximum density of the donor is,

$$
\rho_{d}^{\operatorname{cgs}}=\rho_{d}^{\max }\left[\frac{M_{\mathrm{cgs}}}{M_{\mathrm{d}}+M_{\mathrm{a}}}\right]\left(\frac{a_{\mathrm{cgs}}}{a_{0}}\right)^{-3}=\frac{\rho_{d}^{\max }}{\Omega_{0}^{2}}\left[\frac{4 \pi^{2}}{P^{2} G}\right]_{\mathrm{cgs}}=68.1\left[\frac{3 \pi}{P^{2} G}\right]_{\mathrm{cgs}} .
$$

If, instead, the configuration's reduced mass $\mu_{\mathrm{cgs}}=M_{\mathrm{cgs}}\left[q_{0} /\left(1+q_{0}\right)^{2}\right]$ and orbital frequency $\Omega_{\mathrm{cgs}}=$ $2 \pi / P_{\text {cgs }}$ are specified in cgs units, then the system's total angular momentum in cgs units is,

$$
J_{\text {cgs }}=J_{\text {tot }}\left[\frac{\Omega_{0}}{\left(M_{a}+M_{d}\right)^{5}}\right]^{1 / 3}\left[\frac{\left(1+q_{0}\right)^{2}}{q_{0}}\right]^{5 / 3}\left[\frac{G^{2} \mu^{5}}{\Omega}\right]_{\mathrm{cgs}}^{1 / 3}=2.82\left[\frac{G^{2} \mu^{5}}{\Omega}\right]_{\mathrm{cgs}}^{1 / 3} .
$$

The leading numerical coefficient in the final expression for each one of these relations has been obtained by plugging in values of $\Omega_{0}, a_{0}, V_{a}, M_{d}$, etc. drawn from Table 1 . The numerical value of each of these leading coefficients must be reevaluated using the dimensionless parameter values drawn from Table 4 or Table 5 if one wants to scale our Q1.3 or Q0.5 models, respectively, to cgs units.

Note that no mass-radius relationship, or equivalently value for the polytropic constant $K_{\text {cgs }}$, has been assumed above, and thus the polytropic binary model can represent arbitrary types of component stars. One is free to choose any total mass and orbital period, and in most cases the resultant binary model will not represent any realistic type of donor or accretor. However, choosing an orbital period consistent with the mass-radius relationship of a given type of star will yield approximate binary models with components of that type. For example, a Q0.8 model with a total mass of $1.29 M_{\odot}$, can represent equally well a main sequence binary with an orbital period of $\approx 5.4$ hours, or a white dwarf binary with a period of $\approx 70$ seconds.

\section{Implementation of Driving Mechanisms}

In the initial Q1.3 and Q0.5 models (see Figure 1 and Tables 4 and 5) the donor star slightly underfilled its Roche lobe and had to be driven into contact to initiate mass transfer. As has been outlined in the introductory paragraphs of $\S 5$, we tested two driving mechanisms to initiate Roche lobe overflow. Here we describe specifically how these were implemented in the hydrodynamic code. 


\section{C.1. Drag}

During the first 2-3 orbits of evolutions Q1.3-D and Q0.5-D we extracted angular momentum from the binary at a constant rate. This was accomplished by adding a "sink" term $\left(s_{\text {drag }}\right)$ to the

azimuthal component of the equation of motion. Specifically, the right-hand-side of Eq. (15) was modified to give,

$$
\frac{\partial A}{\partial t}+\nabla \cdot(A \boldsymbol{v})=-\frac{\partial p}{\partial \phi}-\rho \frac{\partial \Phi}{\partial \phi}-2 \Omega_{0} S R-\rho R\left[a_{\theta, \mathrm{com}}+2 \Omega_{0} v_{R, \mathrm{com}}\right]-s_{\mathrm{drag}},
$$

where $s_{\text {drag }} \equiv+\alpha_{\text {drag }}\left[\Omega_{0} R^{2} / P_{0}\right] \rho$ and $\alpha_{\text {drag }}$ is a constant that fixes the rate at which specific angular momentum is removed. In evolutions Q1.3-D and Q0.5-D we set $\alpha_{\text {drag }}=0.01$.

For the record, this sink term was incorporated in the hydrodynamics code by effectively adding to the right-hand-side of MTF's Eq. (42) a term of the form,

$$
-\alpha_{\text {drag }}\left[\Omega_{0} R_{i+1 / 2}^{2} / P_{0}\right] \hat{\rho}_{i+1 / 2, j+1 / 2, k}^{(n+a d v e c t i o n)},
$$

where the superscript, subscript, and caret notations are as defined in MTF.

\section{C.2. Expansion}

During the first 2 orbits of evolution Q1.3-E, the specific entropy of the material that was originally in the donor was steadily increased in order to drive a slow expansion of the donor. This was accomplished by adding a source term ( $\left.s_{\text {expand }}\right)$ to Eq. (17) to give,

$$
\frac{\partial \tau}{\partial t}+\nabla \cdot(\tau \boldsymbol{v})=s_{\text {expand }}
$$

where $s_{\text {expand }} \equiv \alpha_{\text {expand }} \tau / P_{0}$ and the expansion coefficient was set to $\alpha_{\text {expand }}=0.01$. Specifically in the hydrodynamics code, after the updates due to the Eulerian transport terms were performed as described by Eq. (39) of MTF, the entropy tracer was further updated according to the finitedifference expression,

$$
\tau_{i+1 / 2, j+1 / 2, k+1 / 2}^{(n+\text { source })}=\tau_{i+1 / 2, j+1 / 2, k+1 / 2}^{(n+\text { advection })}\left[1+\alpha_{\text {expand }} \frac{\Delta t}{P_{0}}\right],
$$

where the superscript and subscript notations are as defined in MTF. Utilizing the hydrocode's ability to track which fluid originally belonged to which star, this update to $\tau$ was applied only to those computational cells in which the fraction of the donor material in that cell was greater than $90 \%$ of the total value.

In order to estimate how a given change in $\tau$ effects the radius of the donor, we note first that for a polytropic gas of index $n=3 / 2$,

$$
\tau=\rho\left[\frac{3}{2} K_{\mathrm{d}}\right]^{3 / 5}
$$


Hence, increasing $\tau$ by a factor of $\left[1+\alpha_{\text {expand }} \Delta t / P_{0}\right]$ effectively increases $K_{\mathrm{d}}$ by a factor of $[1+$ $\left.(5 / 3) \alpha_{\text {expand }} \Delta t / P_{0}\right]$. Furthermore, from the discussion associated with Eq. (3) we see that, for a given mass, the radius of a spherical polytrope of index $3 / 2$ scales linearly with $K$. Setting $\alpha_{\text {expand }}=0.01$ therefore should result in the radius of the donor expanding at a rate of $1.67 \%$ per orbit.

\section{REFERENCES}

Blondin, J. M., Richards, M. T., \& Malinowski, M. L. 1995, ApJ, 445, 939

Chandrasekhar, S. 1958, An Introduction to the Study of Stellar Structure, (New York: Dover Publications)

Eggleton, P. P. 1983, ApJ, 268, 368

Frank, J., King, A. R., \& Raine, D. J. 2002, Accretion Power in Astrophysics (3d ed; Cambridge: Cambridge Univ. Press)

Gokhale, V., Peng, X. \& Frank, J. 2005, in preparation

Hachisu, I. 1986, ApJS, 62, 461

Hachisu, I., Eriguchi, Y. \& Nomoto, K. 1986, ApJ, 311, 214

Han, Z. \& Webbink, R. F. 1999, A\&A 349, L17

Hils, D. \& Bender, P. L. 2000, ApJ 537, 334

Hjellming, M. S. \& Webbink, R. F. 1987, ApJ 318, 794

King, A. R. 1988, QJ 29, 1

Lai, D., Rasio, F. A., \& Shapiro, S. L. 1993a, ApJ, 406, 63

Lai, D., Rasio, F. A., \& Shapiro, S. L. 1993b, ApJS, 88, 205

Lai, D., Rasio, F. A., \& Shapiro, S. L. 1994a, ApJ, 420, 811

Lai, D., Rasio, F. A., \& Shapiro, S. L. 1994b, ApJ, 423, 344

Lai, D., Rasio, F. A., \& Shapiro, S. L. 1994c, ApJ, 437, 742

Landau, L.D. \& Lifschitz, E.M. 1959, Fluid Mechanics, (New York: Pergamon Press)

Marsh, T. R. \& Steeghs, D. 2002, MNRAS 331, L7

Marsh, T. R., Neelemans, G. \& Steeghs, D. 2004, MNRAS 350, 113

Motl, P. M., Tohline, J. E. \& Frank, J. 2002, ApJS, 138, 121 (MTF) 
Motl, P. M., D’Souza, M. C. R., Tohline, J. E. \& Frank, J. (2005) in preparation

Nelemans, G., Portegies Zwart, S. F., Verbunt, F. \& Yungelson, L. R. 2001, A\& A, 368, 939

New, K. C. B. \& Tohline, J. E. 1997, ApJ, 490, 311

Paczyński, B. 1971, Ann.Rev.Ast.Ap. 9, 183

Patterson, J., Halpern, J., \& Shambrook, A. 1993, ApJ, 419, 803

Rappaport, S., Joss, P.C. \& Webbink, R. F. 1982, ApJ 254, 616

Rasio, F. A. \& Shapiro, S. L. 1992, ApJ, 401, 226 (RS92)

Rasio, F. A. \& Shapiro, S. L. 1994, ApJ, 432, 242 (RS94)

Rasio, F. A. \& Shapiro, S. L. 1995, ApJ, 438, 887 (RS95)

Soberman, G. E., Phinney, E. S. \& van den Heuvel, E. P. J. 1997, A\&A 327, 620

Swesty, F. D., Wang, E. Y. M. \& Calder, A. C. 2000, ApJ, 541, 937

Tutukov, A.V. \& Yungelson, L.R. 1996, MNRAS 280, 1035

Uryu, K. \& Eriguchi, Y. 1998a, MNRAS 296, L1

Uryu, K. \& Eriguchi, Y. 1998b, ApJS118, 563

Verbunt, F. \& Rappaport, S. 1988, ApJ 332, 193

Webbink, R. F., \& Iben, I., Jr. 1987, Proceedings of the $2^{\text {nd }}$ Conference on Faint Blue Stars, p. 445 


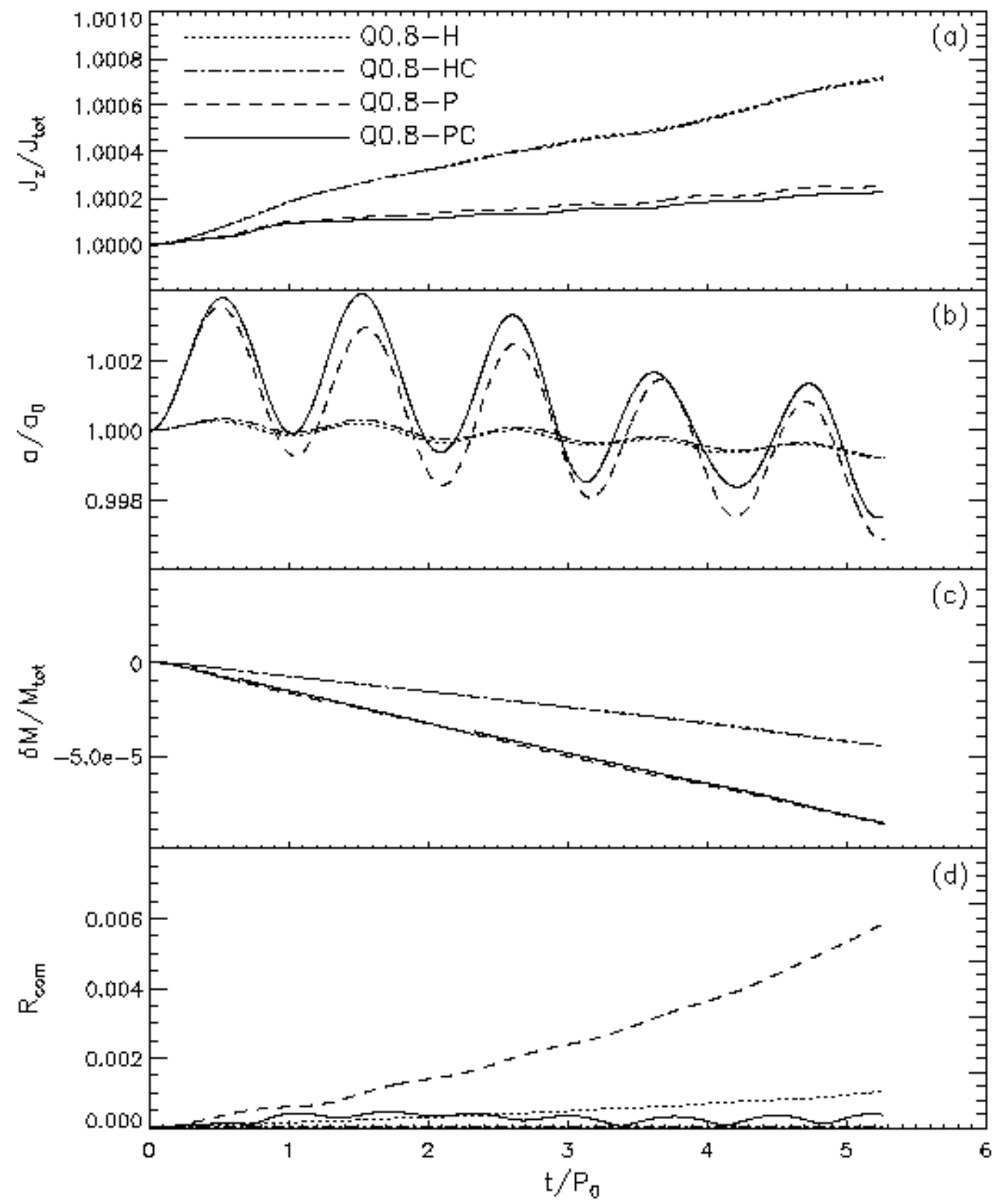

Fig. 2.- Results from the evolution of model Q0.8 using four slightly different versions of the hydrocode (evolution $\mathrm{H}$ - dotted curve; $\mathrm{HC}$ - dash-dot curve; $\mathrm{P}$ - dashed curve; $\mathrm{PC}$ - solid curve). From top to bottom: (a) The $z$-component of the system's angular momentum $J_{z}$, normalized to its initial value $J_{\text {tot }}$, is plotted as a function of time $t$, normalized to the system's initial orbital period $P_{0}=2 \pi / \Omega_{0}$. (b) The binary separation $a$, normalized to its initial value $a_{0}$, is plotted as a function of $t / P_{0}$. (c) The difference between the total mass of the binary system and its initial value, $\delta M \equiv M(t)-M_{\text {tot }}$, normalized to the initial mass, is plotted as a function of $t / P_{0}$. (d) The distance in code units $R_{\text {com }}$ from the axis of the grid to the instantaneous center of mass as a function of $t / P_{0}$. Note that the boundary of the innermost radial cell in these units is at $R_{\text {com }}=0.008$. 

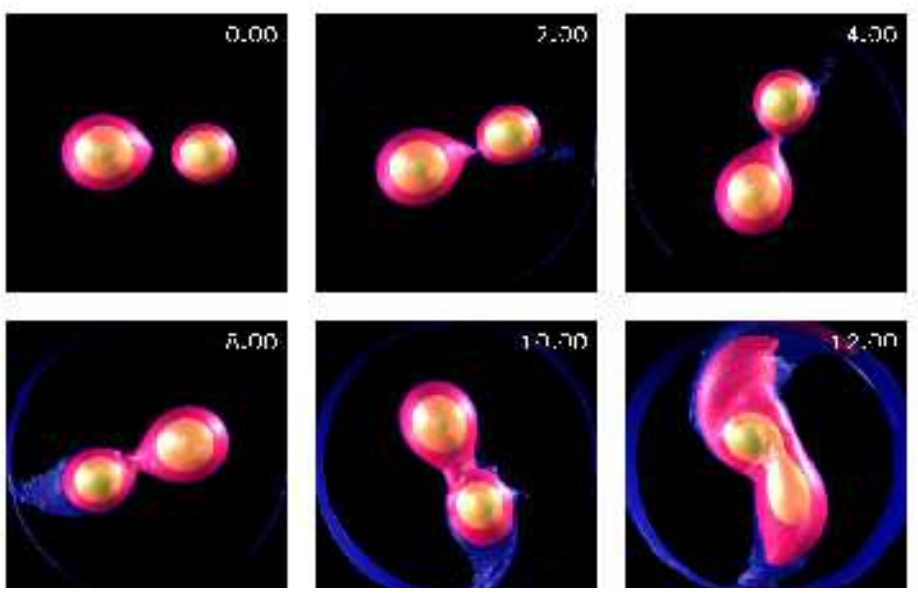
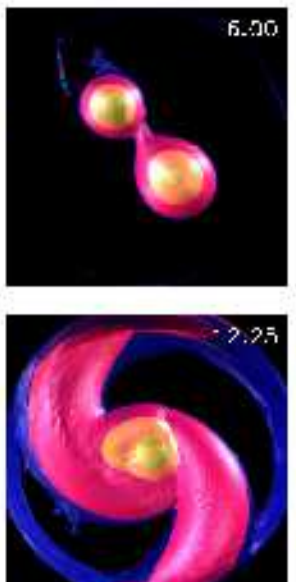

Fig. 3.- A three-dimensional rendering of the mass density at selected time slices during the Q1.3-D evolution that demonstrate direct impact accretion. Times are shown on the top right of each frame in units of the initial orbital period. Each image is a snapshot as viewed by an observer looking down on the equatorial plane while rotating with the angular velocity $\Omega_{0}$. The four colored transparent shells are iso-density surfaces shown at levels $\rho / \rho_{\mathrm{a}}^{\max }=0.5$ (green), 0.1 (yellow), $10^{-3}$ (red) and $10^{-5}$ (blue) where $\rho_{\mathrm{a}}^{\max }$ is the maximum density of the accretor - the star on the right in the initial frame $(t=0)$ of the figure. In the online version this figure is supplemented by a mpeg animation showing the full evolution. 


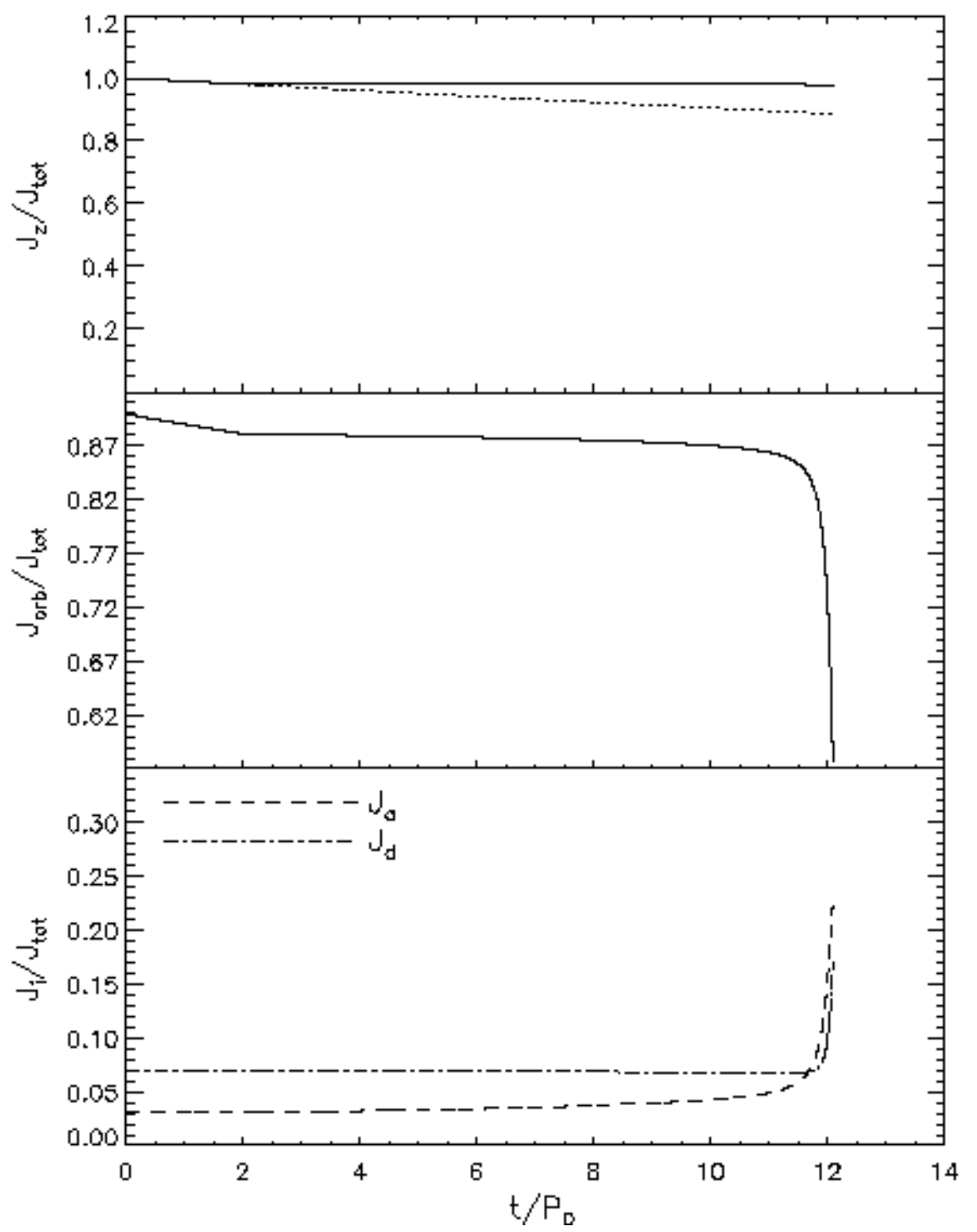

Fig. 4.- Top: The $z$-component of the total angular momentum with respect to an inertial frame centered at the center of mass, $J_{z}$, normalized to its initial value for the Q1.3-D (solid curve). The initial loss rate of angular momentum at $1 \%$ per orbit is denoted by the dotted line. The driving for Q1.3-D is stopped after two orbits and this can be seen when the solid curve becomes horizontal. Middle and bottom: evolution of the orbital angular momentum and the spin angular momenta of the components all normalized to the total initial angular momentum for the Q1.3-D run. 


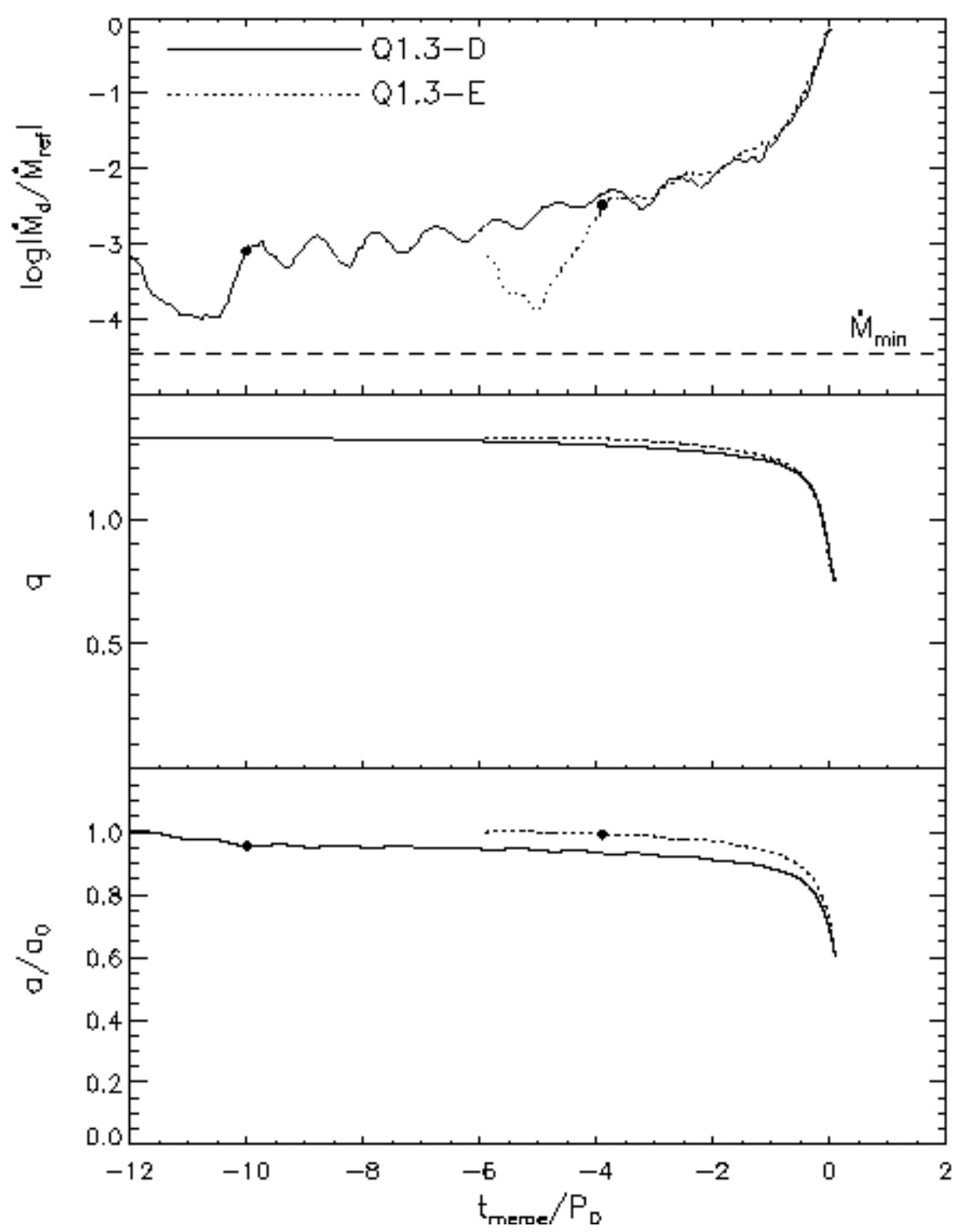

Fig. 5. - The evolution of the mass-transfer rate (top), the mass-ratio $q$, and the separation $a$ for the same initial binary driven to contact by removing angular momentum (Q1.3-D, solid curves) and by expansion of the donor (Q1.3-E, dotted curves). The times shown are times to 'merger' measured in initial binary periods. The dashed line in the top panel indicates the minimum level of mass transfer $\dot{M}_{\text {min }}$ that is resolvable in our simulations (see text). The separation $a$ is normalized to its initial value $a_{0}$. The mass transfer rate is normalized to the reference value $\dot{M}_{\text {ref }} \equiv M_{\mathrm{d}}(0) / P_{0}$ (initial donor mass divided by initial orbital period). For most of the evolution $q>1$, so $a$ decreases as a result of mass transfer even in the absence of driving. The dots mark the points at which driving was stopped. 

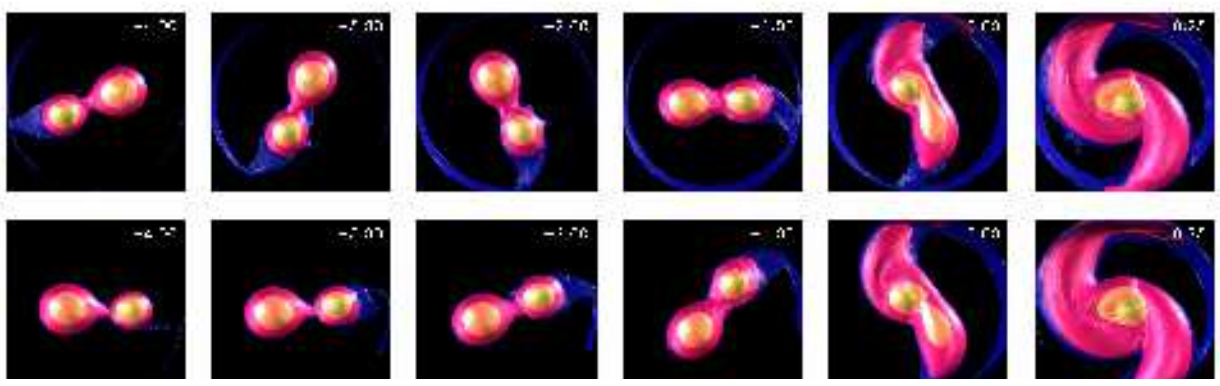

Fig. 6. - Two evolutions with $q_{0}=1.3$, in which mass transfer is initiated by angular momentum losses (Q1.3-D, top row) and by expansion of the donor (Q1.3-E, bottom row), lead to an almost identical merger. The entire Q1.3-E evolution is depicted in a mpeg animation in the online version of this article. The time shown in the movie is $t_{\mathrm{E}}$.
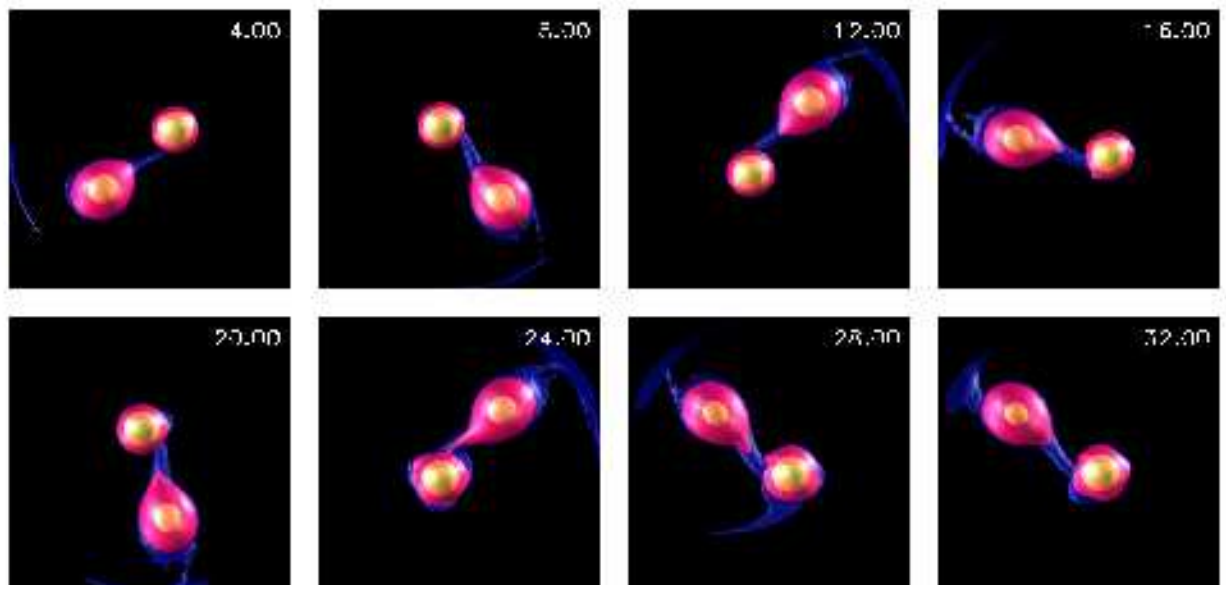

Fig. 7.- Same as Figure 3, but for our Q0.5-Da evolution; images are separated in time by four initial orbital periods. Even after 32 orbits, the system continues to undergo mass transfer and there is no indication that the system will merge. The entire evolution is depicted in the mpeg animation accompanying the online article. 


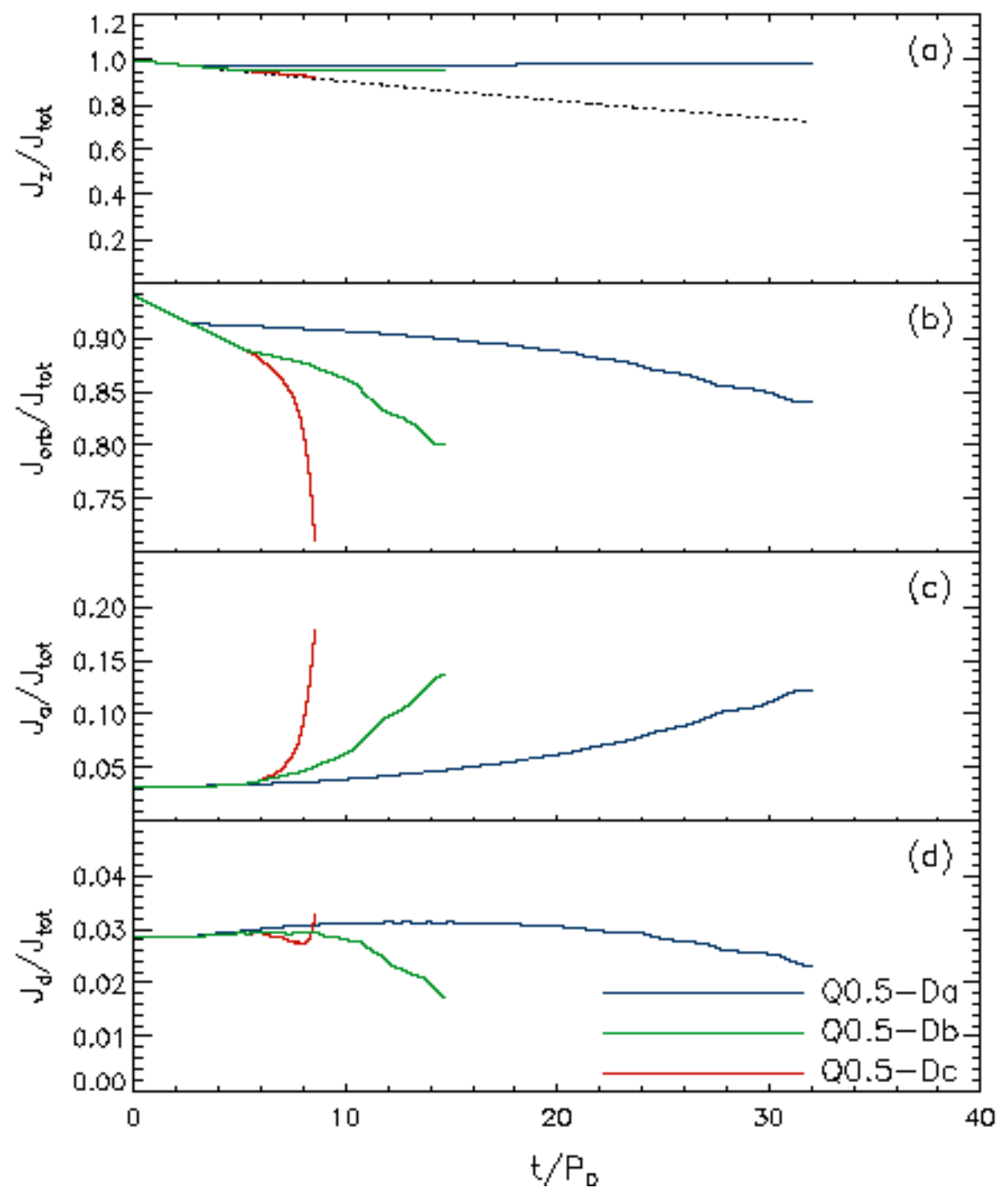

Fig. 8.- The same quantities as in Figure 4, but for the Q0.5-D evolutions: Q0.5-Da (blue), Q0.5-Db (green), and Q0.5-Dc (red). Note that the vertical scale for panel (d) showing $J_{\mathrm{d}} / J_{\text {tot }}$ has been expanded for clarity. 


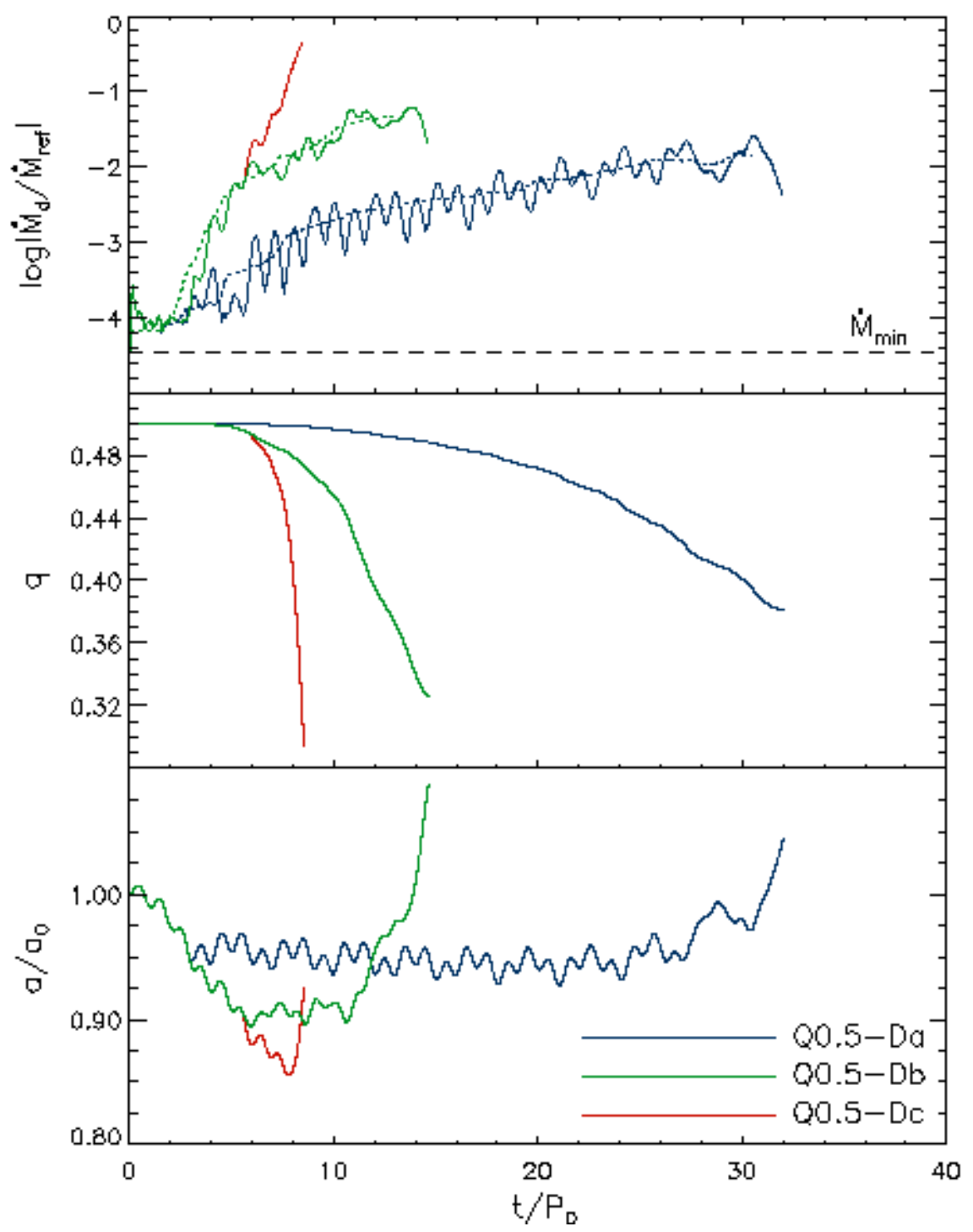

Fig. 9.- Same as Figure 5 but for the Q0.5-D evolutions: Q0.5-Da (blue), Q0.5-Db (green), and Q0.5-Dc (red). All quantities are plotted versus the evolutionary time measured in units of the initial orbital period of the system. For evolutions Q0.5-Da and Q0.5-Db we also show in the top panel, as dashed lines, a 3-orbit boxcar average of the mass transfer rate. 

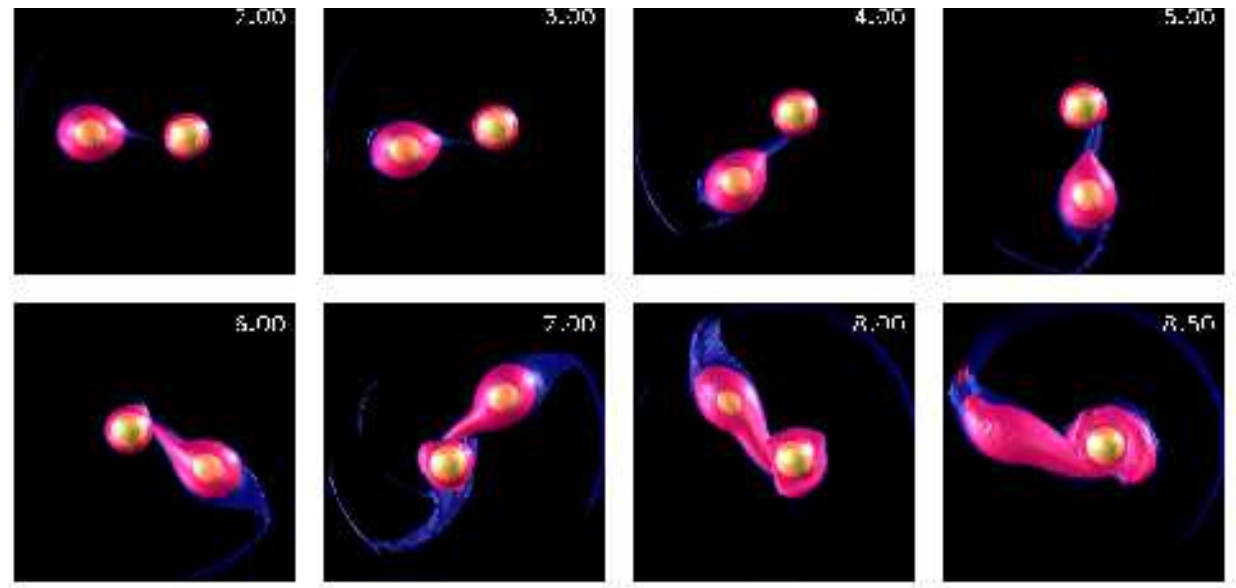

Fig. 10.- Same as Figure 7, but for our Q0.5-Dc evolution; images are separated in time by one initial orbital period, except the last image. Since this binary is continuously driven, the separation and the orbital period decrease throughout, except perhaps at the very end. 


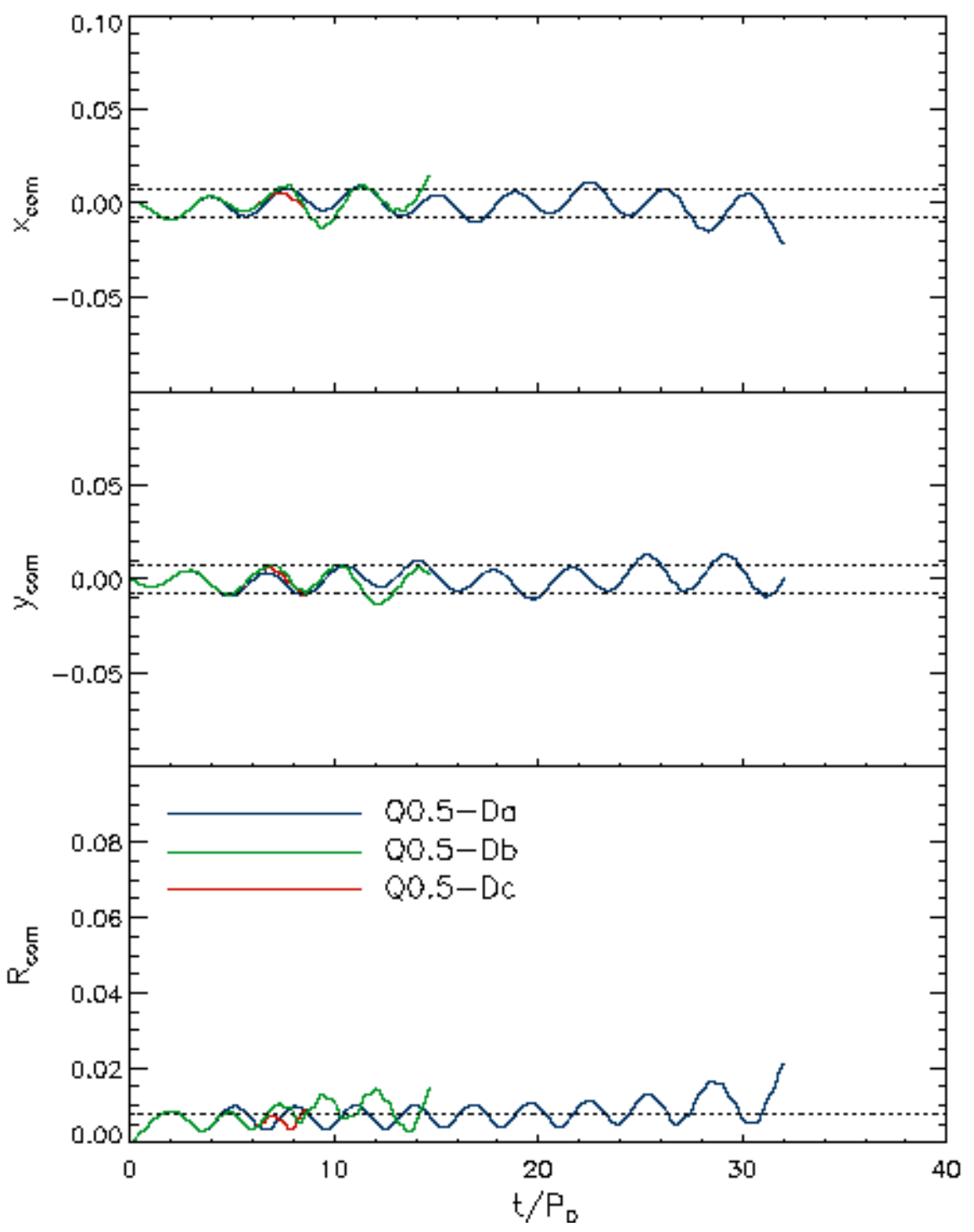

Fig. 11.- Drift of the center of mass during the Q0.5-D simulations: Q0.5-Da (blue), Q0.5-Db (green), and Q0.5-Dc (red). From top to bottom: the $x$ and $y$ components of the position of the center of mass in the corotating frame, and the cylindrical radial distance to the center of mass $R_{\text {com }}$, as functions of $t / P_{0}$. The dotted lines at $\pm \Delta R$ show the extent of the innermost radial grid zone. The center of mass stays very nearly within this one radial grid zone throughout these evolutions. 